
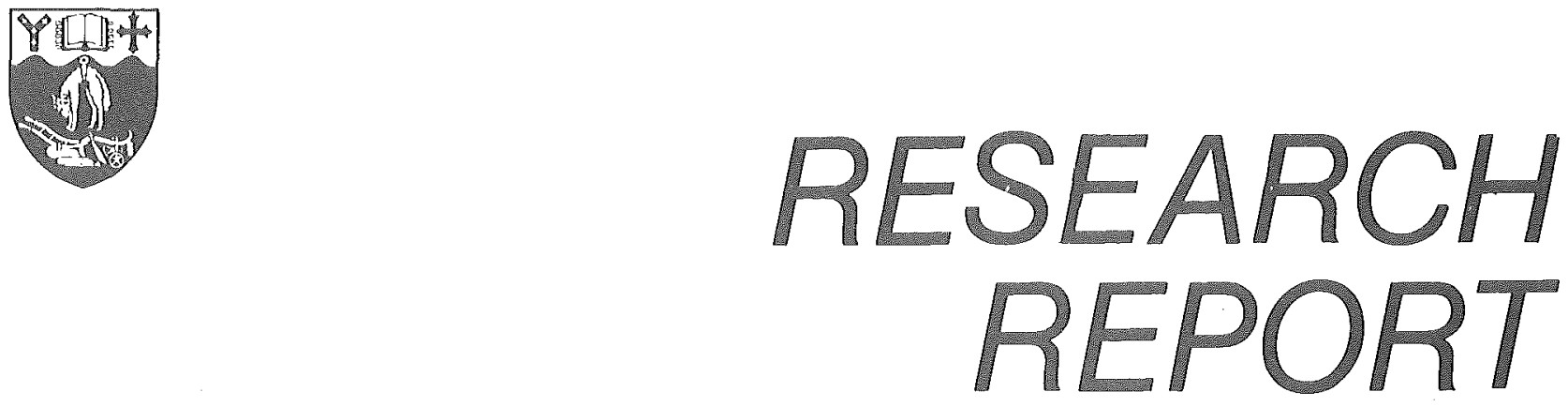

\title{
STAR GAZING IN AFFINE PLANES
}

\author{
D. R. Breach
}

No. 17

August 1981

Reprinted December 1981

Department of Mathematics

University of Canterbury

Christchurch

New Zealand 
STAR GAZING IN AFFINE PLANES

D.R. Breach

No. 17

August 1981

Reprinted December 1981

This report is the text of an invited address given at the Ninth Australian Conference on Combinatorial Mathematics, the University of Queensland, Brisbane Queensland, Australia; August 1981.

This reprinting has allowed the correction of some minor misprints in the original and also for the redrawing of some of the diagrams; December 1981 . 
STAR GAZING IN AFFINE PLANES

It is however better to assume as a postulate the fact, inseparably connected with the idea of a straight line, that there exists only one straight line containing two given points, or, if two straight lines have two points in common, they coincide throughout.

Sir Thomas L. Heath commenting on Euclid's postulate 1, (1908).

\section{THE AUTHOR'S COMPLAINT}

Even in the study of modern geometry it is as well to remember that geometry has its origin in the measurement of the earth, that is to say surveying, and that figures and diagrams are the very heart of the subject both for the transmission and preservation of information and for the development of new ideas and methods. Therefore, while accepting that a diagram in itself is not a proof, one should not be reluctant to introduce them into one's formal presentations. After all, if a figure has been helpful to you in composing your thoughts why selfishly deny that figure to others?

In teaching a course on combinatorics I have found students doubting the existence of a finite projective plane geometry with thirteen points on the grounds that they could not draw it (with 'straight' lines) on paper although they had tried to do so. Such a lack of appreciation of the spirit of the subject is but a consequence of the elements of formal geometry no longer being taught in undergraduate courses. Yet these students were demanding the best proof of existence, namely, production of the object described.

It seems to me that finite projective planes are not good objects to draw convincingly but affine planes are, in that lines which are technically parallel can be made visibly parallel at the cost of drawing them piecewise in straight segments. It may have been serendipity that the first case I tried worked out so nicely and it may have been just coincidence that the resulting figures were enough to activate an interest in finite inversive planes, their construction from finite affine planes and their interrelationship with t-designs, but I now shamelessly present a fig.laden dissertation on finite affine planes and circle geometries. 
But first some formalities.

\section{FINITE PROJECTIVE AND AFFINE PLANES}

$$
\begin{aligned}
& \text { The White Rabbit put on his spectacles. "Where shall } \\
& \text { I begin, please your Majesty?" he asked. } \\
& \text { "Begin at the beginning," said the King, very gravely, } \\
& \text { "and go on till you come to the end: then stop." } \\
& \text { Lewis Carroll, Alice's Adventures } \\
& \text { in Wonderland. }
\end{aligned}
$$

The customary modern beginning of a discourse on finite geometries is to embody the relationships between two kinds of things, points and lines, into a set of axioms and then to deduce consequences therefrom. But please remember that these rather austere axioms are not divorced from reality but come from an attempt to isolate for concentrated study a single aspect, in this case incidence, of that corpus of knowledge and observation which we know to be geometry.

Here is a set of axioms for a plane projective geometry:

Al: Every two distinct points lie on a unique line.

A2: Every two distinct lines lie on a unique point.

A3: There are four distinct points no three of which lie on the same line.

To guarantee a finite projective plane geometry there must be added to these axioms the assumption that:

There is for some $n \geqslant 2$ a line with exactly $n+1$ points. It then follows (e.g. see Hughes and Piper [8]) that a finite projective plane has $n^{2}+n+1$ points in all with exactly $n+1$ points per line. Also there are $n^{2}+n+1$ lines with just $n+1$ of them through each point. The integer $\mathrm{n}$ is the order of the geometry. A finite projective plane geometry of order' $n$ will be denoted by $P G(n)$.

From a projective plane any line together with the points on it can be deleted to leave an affine plane. The lines in an affine plane fall into parallel classes. Each parallel class consists of all those lines which passed through a particular point of the line deleted from the projective plane. In an affine plane two lines from the same parallel class never intersect whereas two 
lines from different parallel classes always intersect. The construction applied to a $P G(n)$ produces a finite affine plane of order $n$ denoted by $A G(n)$. An $A G(n)$ has $n^{2}$ points, $n^{2}+n$ lines, and $n+1$ parallel classes of $n$ lines each. The construction of an $A G(n)$ from a $P G(n)$ is reversible in that there is always a unique $P G(n)$ into which a particular $A G(n)$ can be embedded by the addition of a line with $n+1$ points. A PG(n) may give rise to non-isomorphic $A G(n)$ 's depending on the choice of the line to be deleted. (Hughes and Piper, [ 8]). In principle a complete knowledge of $P G(n)$ 's implies a complete knowledge of $A G(n)$ 's and vice-versa.

\section{STAR DIAGRAMS}

Figure 1 is a star diagram of an $A G(4)$; in fact, of the AG(4) since under point permutations it can be shown that all models of $A G(4)$ 's are isomorphic. The dots are the 16 points of the geometry. Other visual line intersections do not correspond to points of the geometry. The lines of the diagram correspond to the lines of the geometry with the understanding that a line may be presented in two or more straight segments drawn parallel to each other. This is clarified by figure 2 which emphasises the four lines belonging to a parallel class of AG(4). The heavy line which appears vertical in this figure really should appear in four parallel segments as in figure 3. However, in constructing these star diagrams for $A G(n)$ 's it is convenient to have the convention that when each point on a line is the sole occupant of its fragment of line then all these fragments shall be turned through a right angle and linked up to form a line that appears perpendicular to all the other lines in the parallel class.

Suppose the points and lines in a finite geometry are labelled. Then an automorphism of the geometry is a point label permutation which takes lines onto lines and preserves incidences. It is usual in the study of geometries to call these automorphisms collineations; however in this work the term automorphism will be used since it is one used also in connection with block designs of which we are about to introduce a special subclass, the t-designs. Figure 4 shows an involution, i.e. an automorphism of order 2 , of AG(4). 


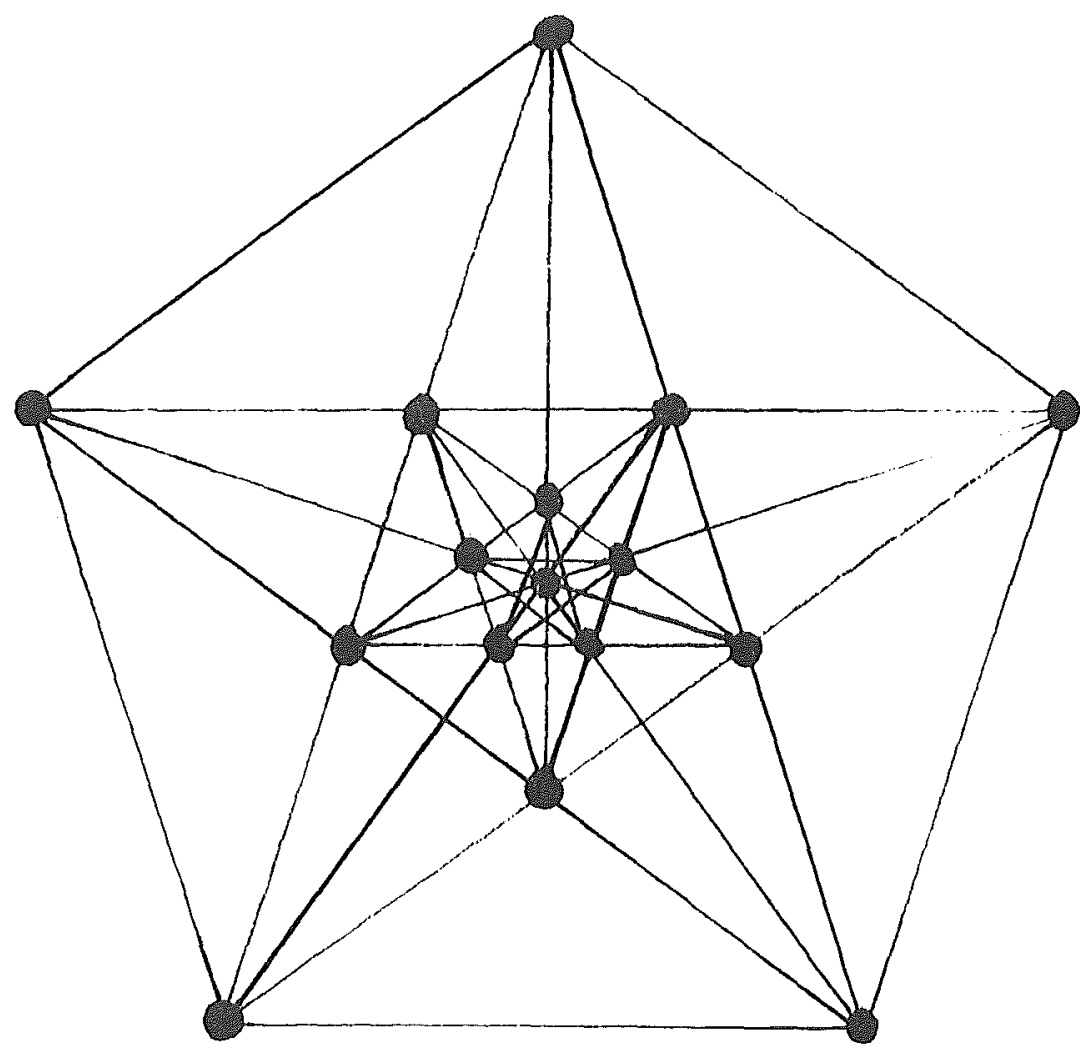
Figure 1. A star diagram of a finite affine plane of order 4 ,
AG(4).

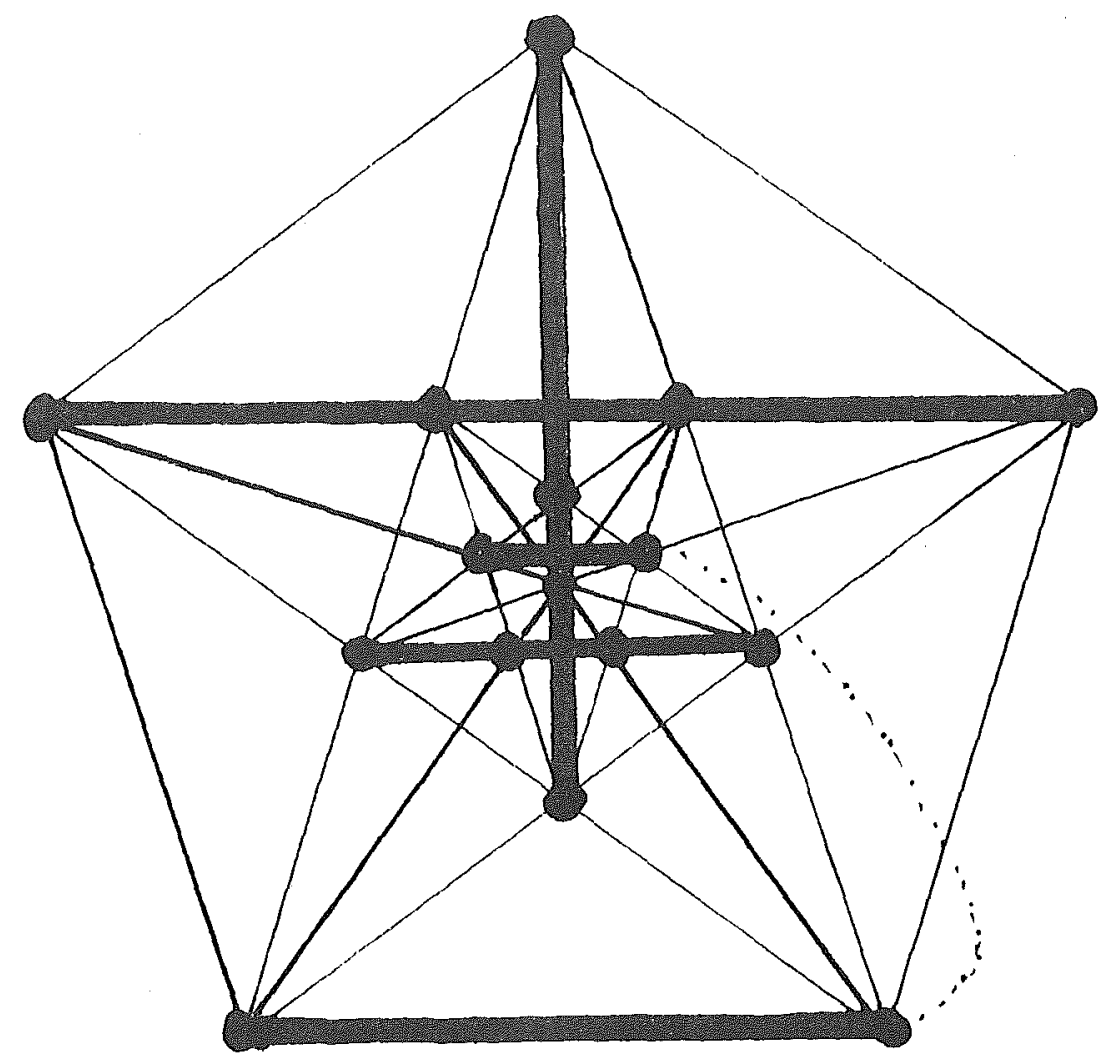

Figure 2. A parallel class in $\mathrm{AG}(4)$. 


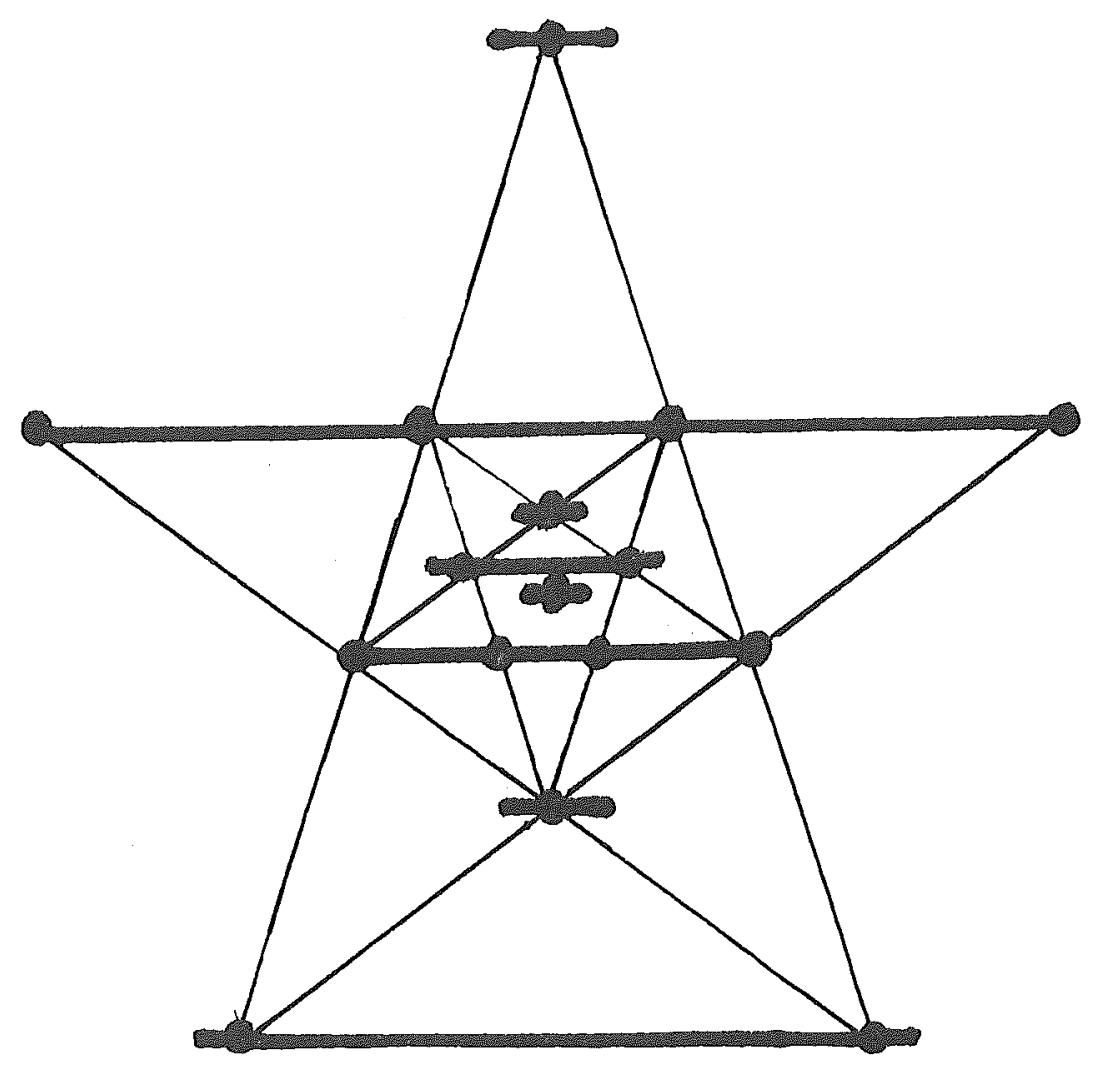

Figure 3. Another way of showing a parallel class in AG(4).

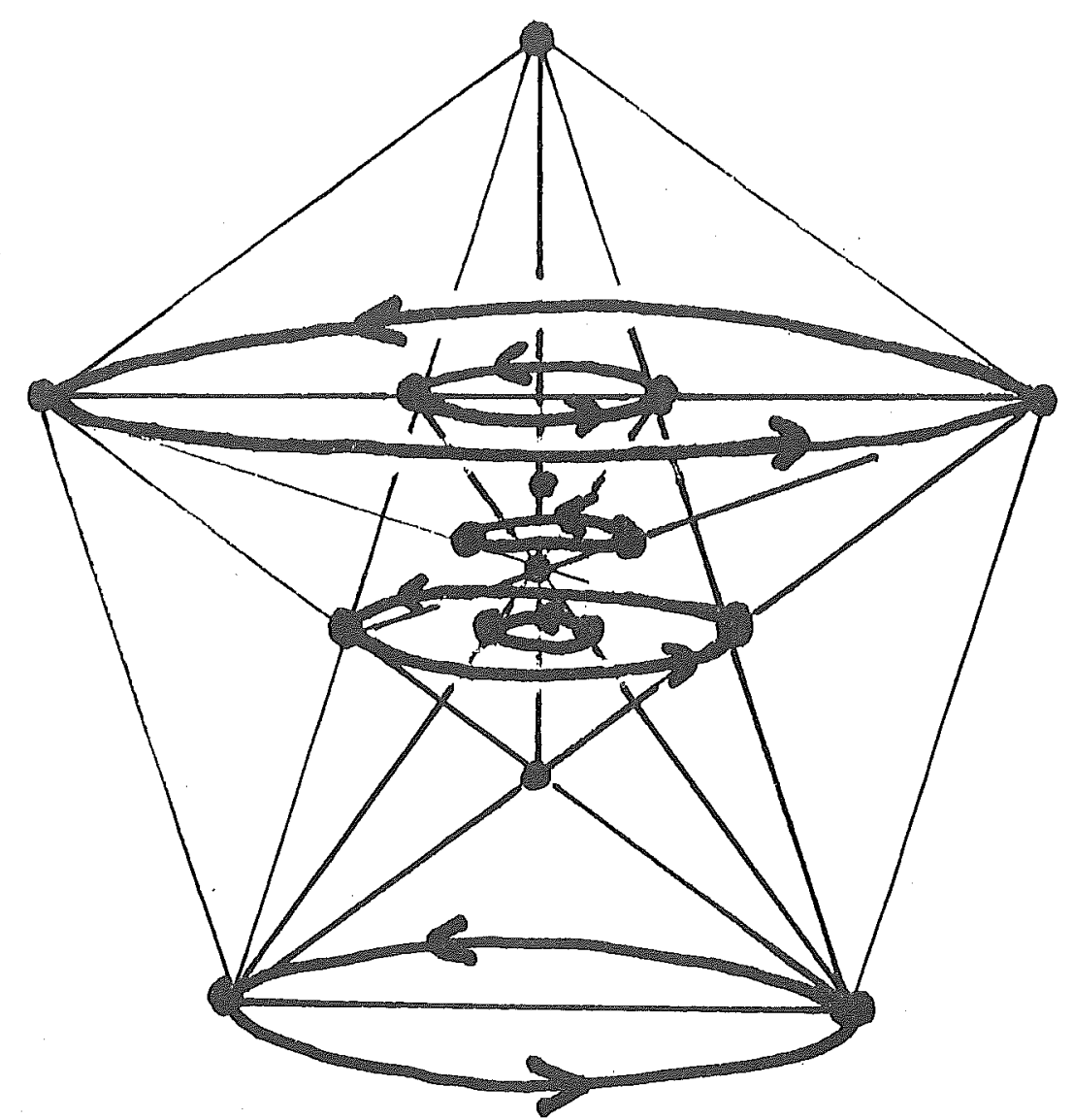

Figure 4. An involution of $\mathrm{AG}(4)$. 
4. FINITE GEOMETRIES AS t-DESIGNS

$\mathrm{A} t-(v, k, \lambda)$ design on a set $\mathrm{S}$ of finite cardinality $\mathrm{v}$ is a collection $D$ of subsets of $S$ with the properties that:

(i) all the subsets, called brocks, have the same cardinality $\mathrm{k}$;

(ii) for $t$ fixed any $t$-subset of $S$ is a subset of exactly $\lambda$ blocks;

(iii) all the blocks are distinct.

The elements of $\mathrm{S}$ are commonly called points. If every one of the possible $\left(\begin{array}{l}\mathrm{v} \\ \mathrm{k}\end{array}\right)$ blocks appears in $\mathrm{D}$ then the design is said to be trivial. Traditional notation has $b$ for the number of blocks in $D$ and $r$ for the number of replications of each point in $D$.

Let $\lambda_{i} ; 0 \leqslant i \leqslant t$, be the number blocks of $D$ containing $a$ given set of $i$ points. Then

$$
\lambda_{i}\left(\begin{array}{l}
k-i \\
t-i
\end{array}\right)=\left(\begin{array}{l}
v-i \\
t-i
\end{array}\right) \lambda \quad ; \quad 0 \leqslant i \leqslant t
$$

In particular $\lambda_{0}=\mathrm{b}$ and $\lambda_{1}=r$. (e.g. see Cameron and van Lint [2], p2). The parameters $\lambda_{i}$ must of course be non-negative integers although the satisfaction of this condition does not guarantee the existence of a design.

The automorphism group, Aut D, D is a permutation group on the points $D$ consisting of all those point permutations which send blocks onto blocks and preserve point-block incidences.

A finite projective plane $P G(n)$ of order $n$ is a $2-\left(n^{2}+n+1, n+1,1\right)$ design; the blocks of the design are taken to be the lines of the plane. A finite affine plane $A G(n)$ is likewise a $2-\left(n^{2}, n, 1\right)$ design.

\section{EXTENSIONS AND RESTRICTIONS}

Given a $t$-design $D$ a $(t-1)$-design $D_{x}$ can always be made from it by rejecting all those blocks of $\mathrm{D}$ not containing the point $\mathrm{x}$ and then deleting $x$ from the remaining blocks. Then $D_{x}$ is $a$ restriction of $\mathrm{D}$ on $\mathrm{x}$. In design theory, however, the interest usually lies in increasing $t$. If a design is obtainable as a restriction on a larger design then the smaller design is extendible 


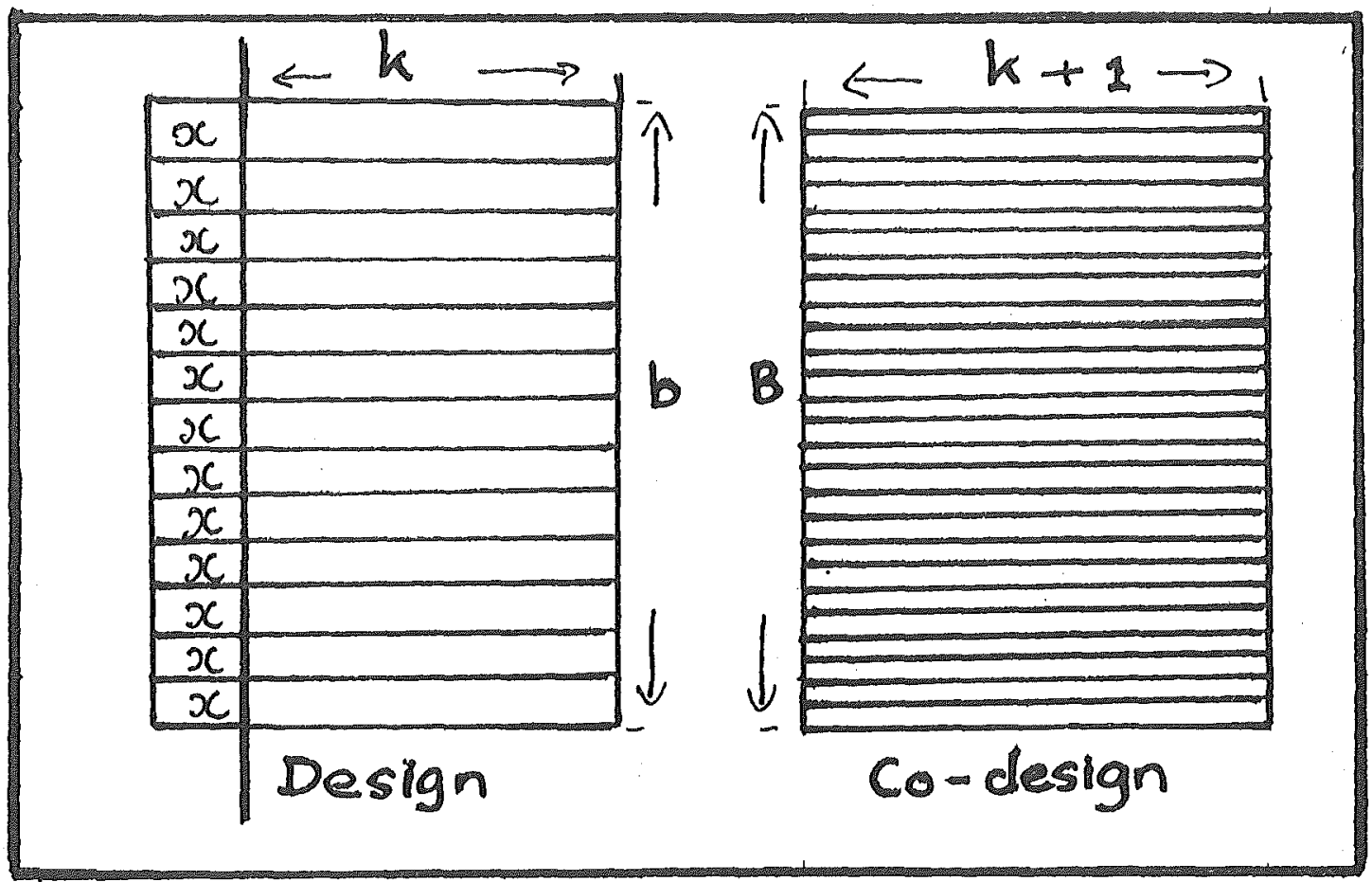

Figure 5. Scheme for extending a $2-(v, k, \lambda)$ design with b blocks by means of a new point $x$ and $a 2-(v, k, \mu)$ co-design with B blocks to produce a 3-design.

to the larger, (which is then an extension of the small design).

Suppose that a $t-(v, k, \lambda)$ design is extendible to a $(t+1)-(v+1, k+1, \lambda)$ design and that $x$ is the extension point. Then in the larger design consider a particular $t$-set of points not containing $x$. This occurs in $\lambda(v-t+1) /(k-t+1)$ blocks, of which $\lambda$ contain $x$. Consequently the blocks which do not contain $\mathrm{x}$ form a $t-(\mathrm{v}, \mathrm{k}+1, \lambda(\mathrm{v}-\mathrm{k}) /(\mathrm{k}-\mathrm{t}+1))$ design. This I call a co-design, a term which may not survive this paper (see figure 5).

A particular model of a $t-(v, k, \lambda)$ design may have more than one co-design, that is to say there may be two or more distinct sets of blocks that extend it to a $(t+1)$-design. These codesigns may or may not be isomorphic. There is a $2-(9,4,3)$ design with three co-designs two of which are isomorphic (Breach [1]). Also it may be possible to repeat the extension process to make $(t+2)$-designs, etc. At present no non-trivial 6-design is known. There are some non-trivial 5-designs (e.g. see Witt [12 1,[13], Pless [11], Denniston [ 6]). One of these, the $5-(12,6,1)$ design, will be recreated later in this paper. 
6. EXTENSIONS OF FINITE PLANES TO CIRCLE GEOMETRIES

The demand that $b$ for a $3-\left(n^{2}+n+2, n+2,1\right)$ design be integral requires $(n+2)$ to divide 12 . Therefore for projective planes we have:

If $P G(n)$ has an extension, then $n=2,4$ or 10.

(Hughes [ 7 ])

In fact the unique $2-(7,3,1)$ design can be extended to a $3-(8,4,1)$ design in just one way. For $n=4$, three successive extensions of the unique $2-(21,5,1)$ design to the unique $5-(24,8,1)$ design can be made. The case $n=10$ is undecided and even the existence of a PG(10) is not known.

It is convenient to call a 3 -design with $\lambda=1$ a circle geometry on the grounds that any three distinct points lie on a unique block. . In this context the blocks are called circles.

For the finite affine planes, $2-\left(n^{2}, n, 1\right)$, the block number $b$ for a possible extension is always an integer so we have

Any $A G(n)$ is a candidate for extension to a 3-design.

The 3-design when it exists belongs to the family of circle geometries known as inversive planes or Möbius planes for which the notation $M(n)$ will be used. Here $n$ is the order of both the inversive plane and the affine plane of which it is an extension.

For $n$ even Dembowski [ 3 ] showed that the inversive planes cannot exist unless $\mathrm{n}$ is a power of 2 and in that case they can always be produced by plane slicing an ovoid in a three dimensional projective space over $G F(n)$. For $n$ odd it is not known whether inversive planes must be ovoidal or not. In this paper examples of ; inversive planes constructed from the 'small' affine planes $(n \leqslant 8)$ will be displayed.

If an inversive plane (or $3-\left(n^{2}+1, n+1,1\right)$ design) is to have an extension to a 4-design or further then from the new block numbers we must have $(n+2)$ dividing 60 . Values of $n$ compatible with Dembowski's result are $2,3,4,8$ and 13. Of these $n=4$ and $\mathrm{n}=8$ have been ruled out by Kantor [9]. Another demonstration of the impossibility of $\mathrm{n}=4$ will be given herein. The case $\mathrm{n}=2$ is trivial. For $n=13$ the matter is undecided. For $n=3$ we have 
the unique $4-(11,5,1)$ and $5-(12,6,1)$ designs associated with the smal1 Mathieu groups $M_{11}$ and $M_{12}$ (Witt, [12], [13]).

The finite Möbius planes $M(n)$ can be distinguished from other circle geometries either by identifying them with the $3-\left(n^{2}+1, n+1,1\right)$ designs as above or by giving three axioms of incidence between points and circles (Dembowski [3], Lüneberg (10)):

Ml. Three distinct points lie on a unique circle.

M2. If $\mathrm{P}$ is a point on the circle $\mathrm{c}$ and $\mathrm{Q}$ is another point not on $C$ then there is a unique circle $C^{\prime}$ through both $\mathrm{P}$ and $\mathrm{Q}$ with $\mathrm{C} \cap \mathrm{C}^{\prime}=\{\mathrm{P}\}$ (i.e. C' touches $c$ at $P$ ).

M3. There are at least two circles and every circle has at least three points.

If one circle contains exactly $(n+1)$ points then all circles do, and there are $\left(n^{2}+1\right)$ points in all on $n\left(n^{2}+1\right)$ circles with $n(n+1)$ circles through each point. The number of circles through both of two distinct points is $(n+1)$. For a given circle there are $\left(n^{2}-1\right)$ others touching it, $\frac{1}{2} n^{2}(n+1)$ cutting it in two points, and $\frac{1}{2} n(n-1)(n-2)$ not intersecting it at all. These statistics can of course be obtained by counting in the 3-design corresponding to $M(n)$.

\section{CONSTRUCTING A 3-DESIGN FROM AG (4).}

First we need a model of $\mathrm{AG}(4)$. A standard construction for $P G(4)$ is to take $\left[\begin{array}{lllll}3 & 6 & 7 & 12 & 14\end{array}\right]$ as a starter block to generate all the other blocks by the transformation $\mathrm{x} \rightarrow \mathrm{x}+1$ (mod 21). Then if the points in the starter block are deleted from the whole design the twenty blocks of $\mathrm{AG}(4)$ remain. For the current discussion it is convenient to relabel the points by the scheme:

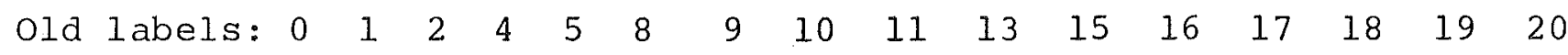

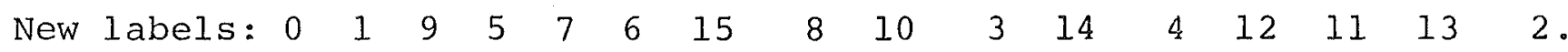
Then the resulting $A G(4)$ can be presented in a star diagram as in figure 6 (some of the line segments have been omitted, cf. fig. 1). There are many star diagrams possible and there is no reason why 0 should have a privileged position at the centre. Figure 7 
is another star diagram of the same $A G(4)$ with 1 at the centre.

To extend to an inversive plane $M(4)$ an extra symbol $\infty$ must be added to each block of the $2-(16,4,1)$ design and a $2-(16,5,4)$ design is needed as a co-design. No block of $M(4)$ can intersect any other in more than two points. Now in figures 6 and 7 (or any other star diagram) the points fall naturally into three concentric circuits of five plus a sixteenth at the centre as in figures 8 and 9 . These circuits turn out to be blocks in the co-design.

In a $P G(n)$ or $A G(n)$ a set of $(n+1)$ points no three of which are collinear is called an oval. In terms of ovals we now formally define a mathematical object corresponding to a star diagram.

Definition: In an $A G(n)$ a star is a collection of $(n-1)$ mutually disjoint ovals each of $(n+1)$ points together with a point not on any of the ovals. This point is called the centre of the star. The order in which the ovals are listed is immaterial.

Figures 8 and 9 illustrate stars centred on 0 and 1 respectively; star A and star B.

Now the $A G(4)$ being used has automorphism permutations

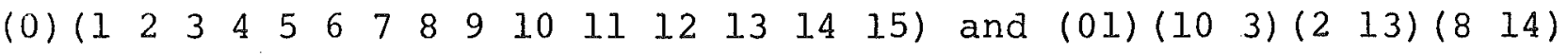
(5 4) (12 15) (6 11) (7 9). There are many others but these are sufficient to show that the design is doubly transitive on points. What is even more important is that the first of these automorphisms not only preserves the $A G(4)$ but also preserves star A by fixing its centre and taking ovals onto ovals. Figure 10 illustrates the orbit of the points. However the same automorphism does not preserve star B but produces from it fifteen different stars with each point other than 0 being at the centre in turn, (figure 11). Thus there are now sixteen stars in all each with three ovals. These 48 ovals are the blocks of the co-design needed to make $M(4)$. We have the 


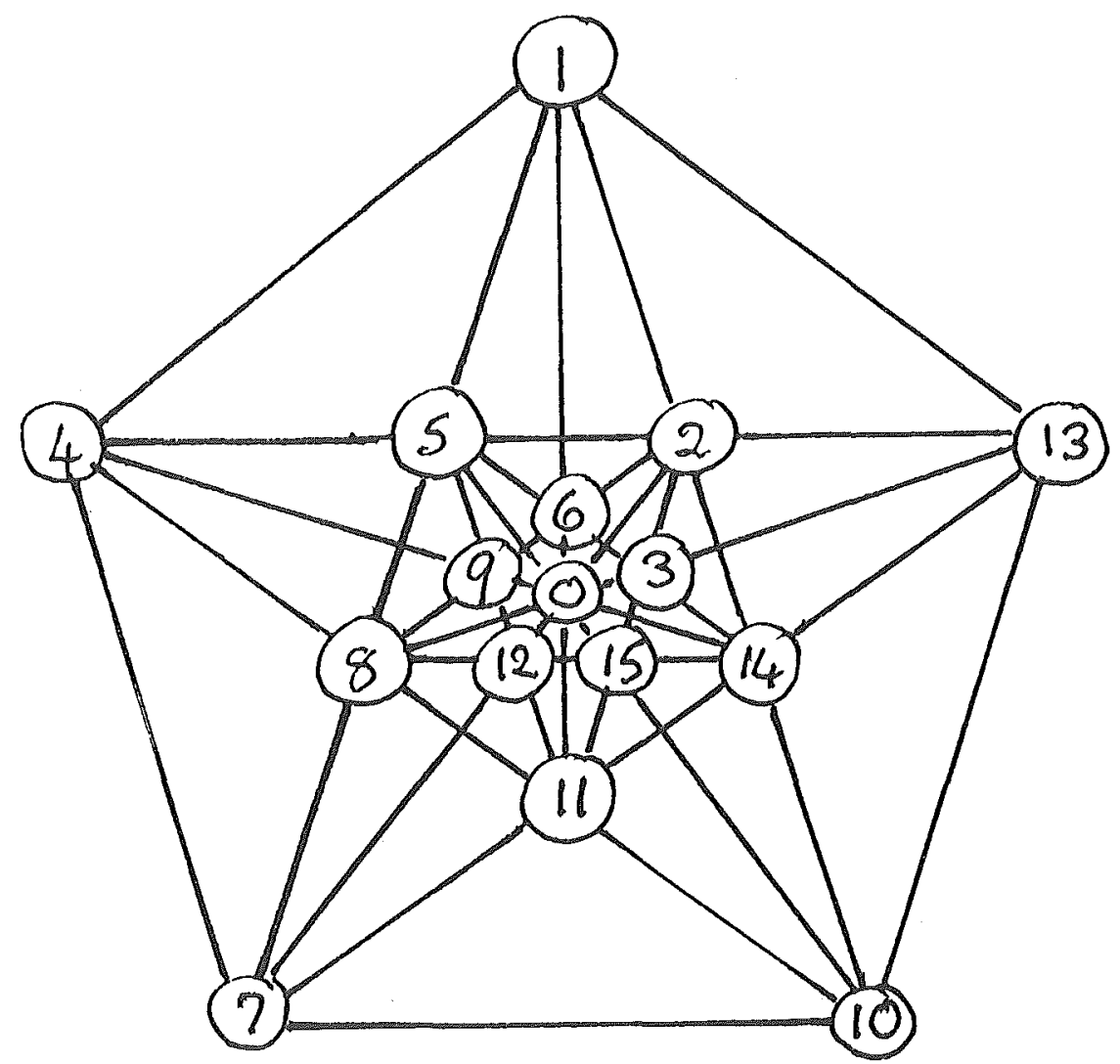

Figure 6. A labelled star diagram of an AG(4) (Star A)

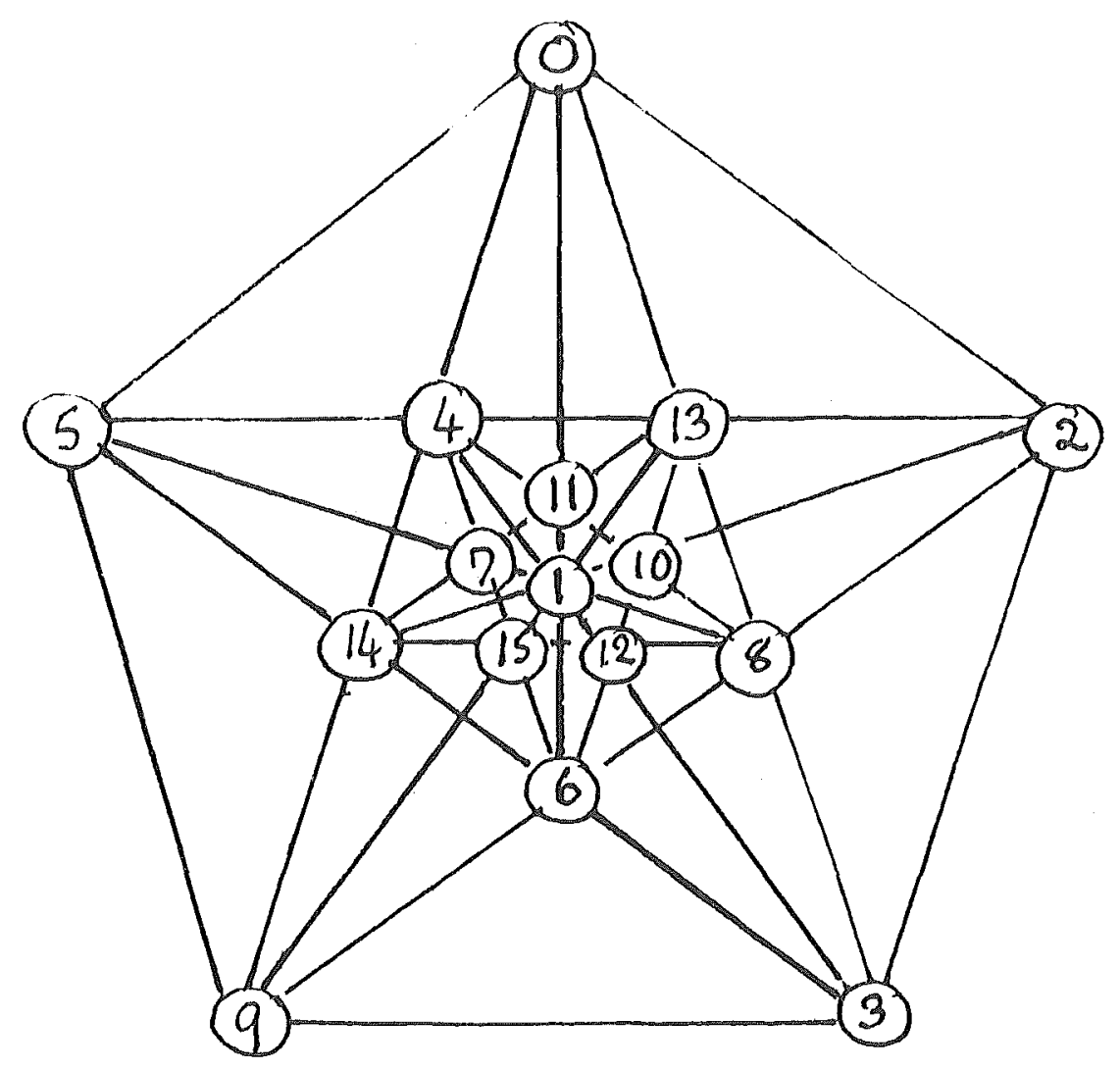

Figure 7. Another labelled star diagram of the same AG(4) (Star B) 


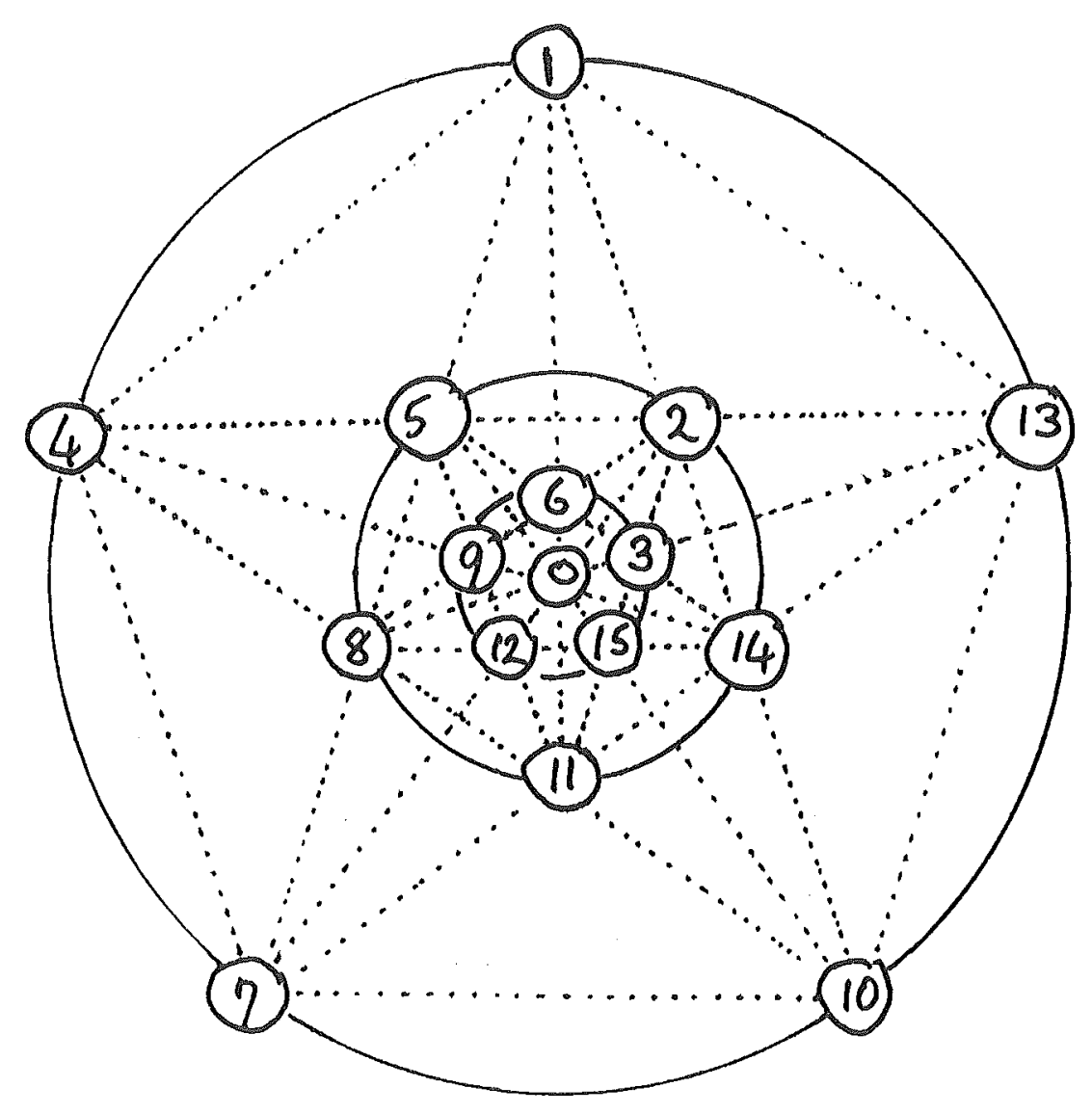

Figure 8. Star A and its ovals

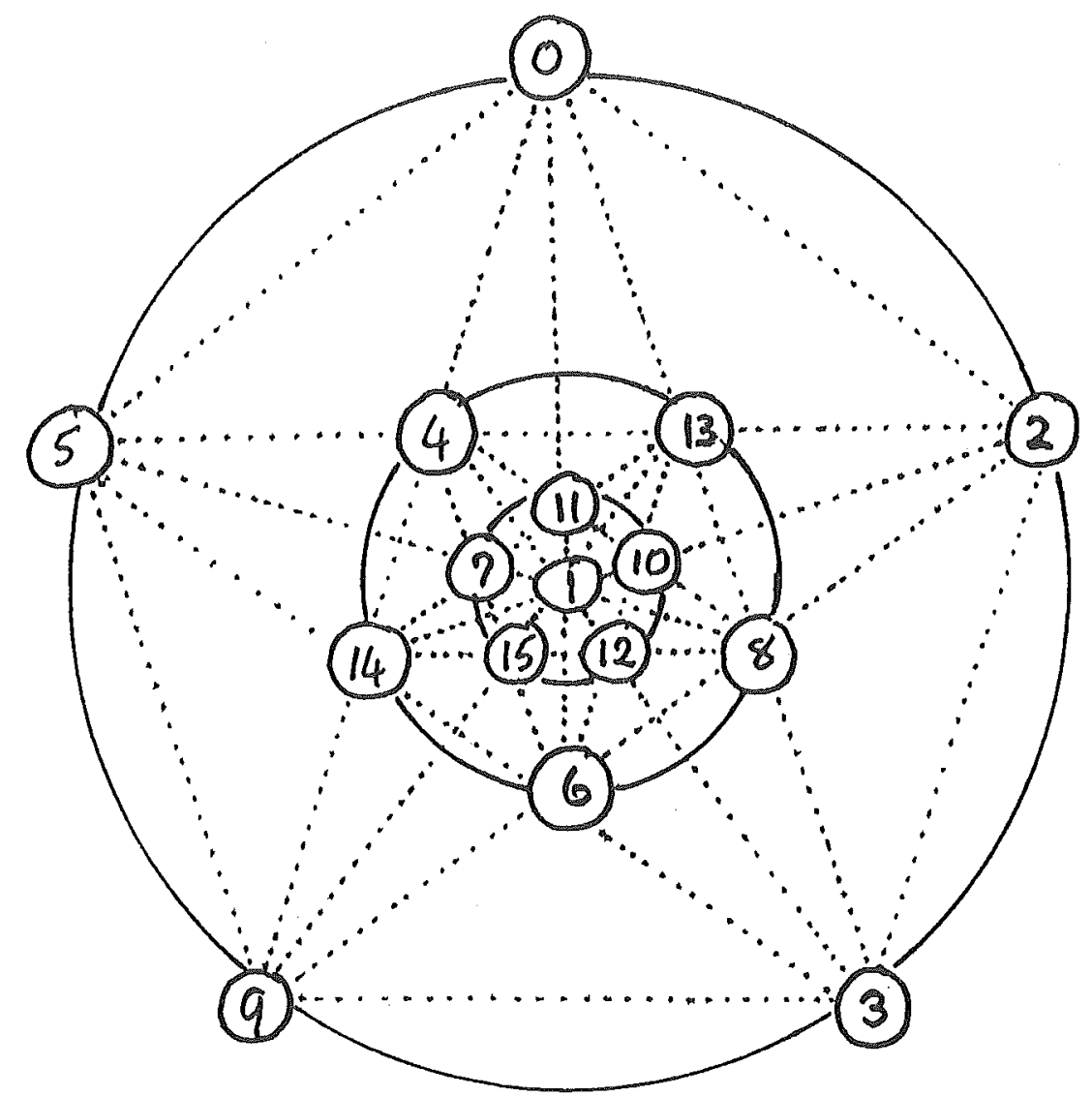

Figure 9. Star $B$ and its ovals 


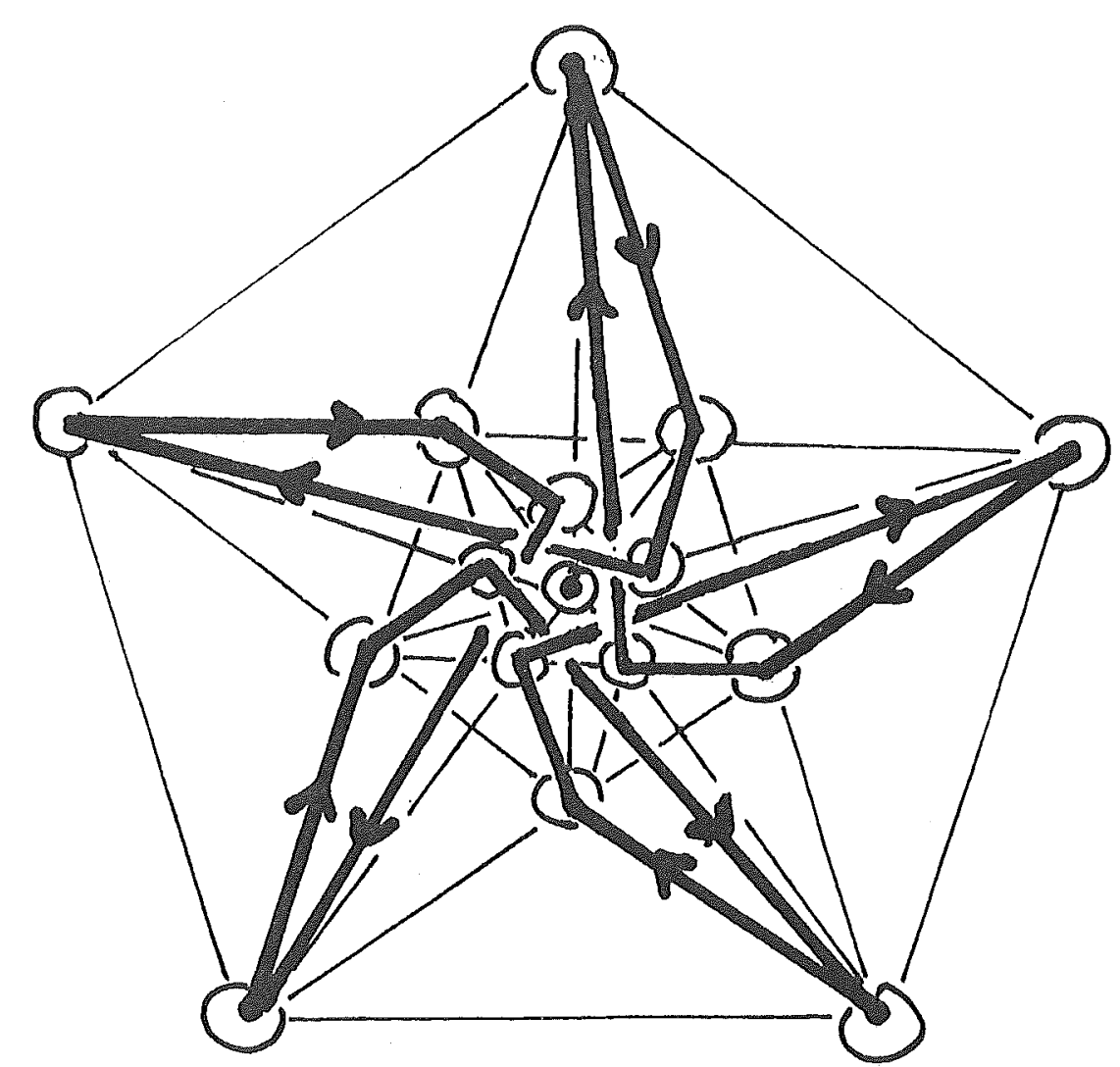

Figure 10. The point orbit in Star A induced by the automorphism (0) $\left(\begin{array}{lllll}1 & 2 & 3 & \ldots & 15\end{array}\right)$

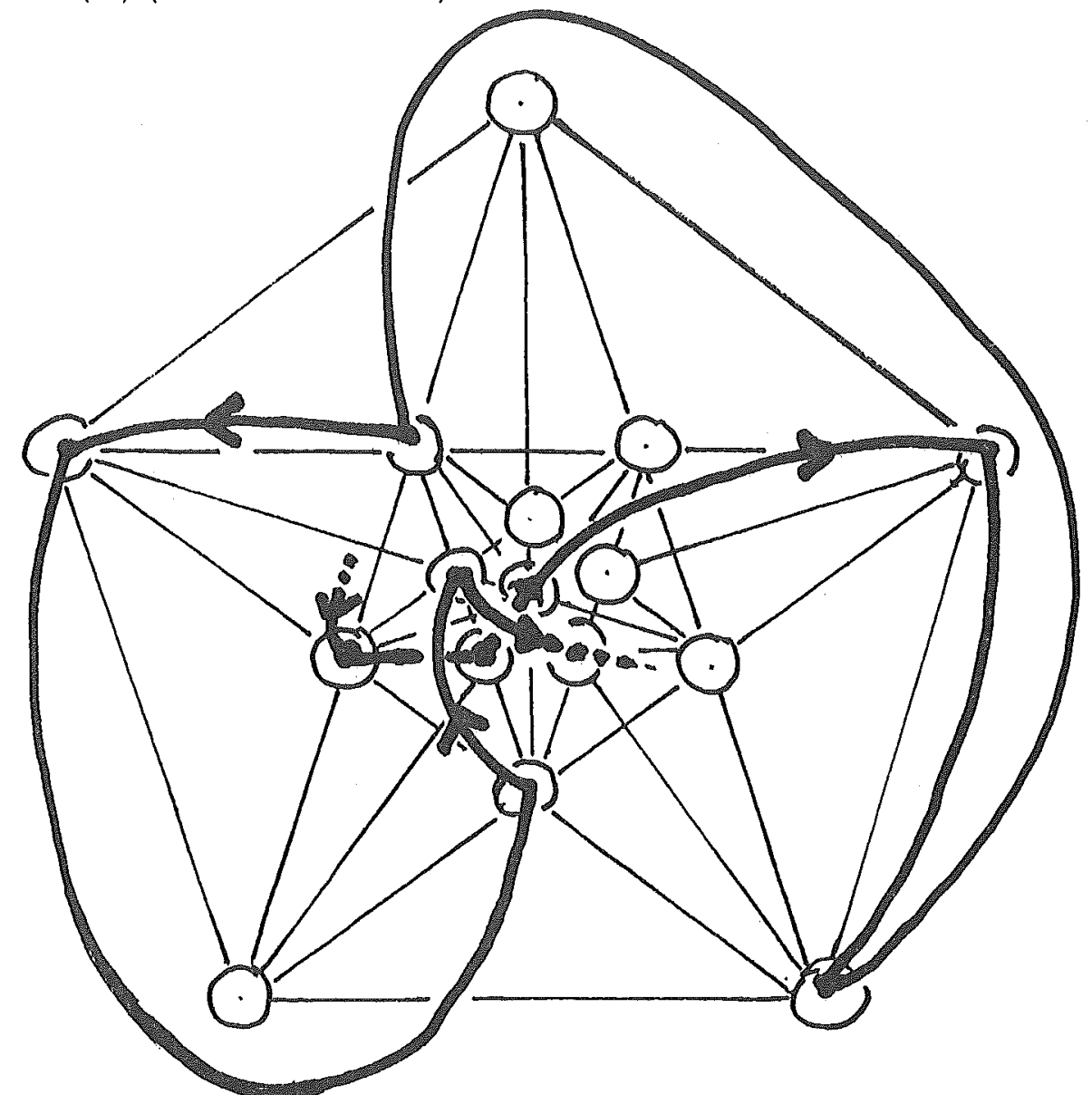

Figure 11. Part of the point orbit in star B induced by (0) $\left(\begin{array}{lllll}1 & 2 & 3 & \ldots & 15\end{array}\right)$ 
Theorem I: The 68 blocks generated by the actions of.

$\alpha: \quad(\infty)(0)\left(\begin{array}{lllllllllllllll}1 & 2 & 3 & 4 & 5 & 6 & 7 & 8 & 9 & 10 & 11 & 12 & 13 & 14 & 15\end{array}\right)$,

and $\beta:\left(\begin{array}{ll}0 & 1\end{array}\right)\left(\begin{array}{lll}10 & 3\end{array}\right)\left(\begin{array}{ll}2 & 13\end{array}\right)\left(\begin{array}{ll}8 & 14\end{array}\right)\left(\begin{array}{ll}5 & 4\end{array}\right)\left(\begin{array}{ll}12 & 15\end{array}\right)\left(\begin{array}{ll}6 & 11\end{array}\right)(7 \quad 9)$,

on the blocks

$\left[\begin{array}{lllll}\infty & 0 & 1 & 6 & 11\end{array}\right], \quad\left[\begin{array}{llllll}1 & 4 & 7 & 10 & 13\end{array}\right]$,

$\left[\begin{array}{lllll}0 & 5 & 9 & 3 & 2\end{array}\right],\left[\begin{array}{lllll}13 & 4 & 14 & 6 & 8\end{array}\right],\left[\begin{array}{lllll}10 & 11 & 7 & 15 & 12\end{array}\right]$,

form a $3-(17,5,1)$ design, i.e. an $\mathrm{M}(4)$.

proof: (i) $\alpha$ and $\beta$ acting on $\left[\begin{array}{lllll}\infty & 0 & 1 & 6 & 11\end{array}\right]$ produce all the 20 blocks in $A G(4)$ with the extension point $\infty$ added to each block.

(ii) $\alpha$ acting on [ $\left[\begin{array}{llllll}1 & 4 & 7 & 10 & 13\end{array}\right]$ produces the three ovals of star $A$ and fixes star A.

(iii) a acting on [ $\left.\begin{array}{lllll}0 & 5 & 9 & 3 & 2\end{array}\right],\left[\begin{array}{lllll}4 & 14 & 6 & 8 & 13\end{array}\right]$, and [ $\left.\begin{array}{llllll}7 & 15 & 12 & 10 & 11\end{array}\right]$, the ovals of star B, and on the centre of B, produces 15 distinct stars all differing from star A.

(iv) No two ovals from star A and star B have more than two points in common.

(v) $\alpha$ and $\beta$ acting together are two-transitive on the 16 stars since $\beta$ interchanges star $A$ and star $B$. Alternatively, introduce a further permutation by swapping the stars underlying the orbit diagrams figures 10 and 11 .

(vi) No oval of any of the 16 stars intersects a block containing $\infty$ in more than two points.

(vii) Therefore no triple of distinct points occurs more than once in the 48 ovals from the stars and the 20 blocks containing $\infty$.

(viii) Calling the ovals blocks the 68 blocks between them contain $68 .\left(\begin{array}{l}5 \\ 3\end{array}\right)=680$ distinct triples. But the number of distinct triples from 17 points is $\left(\begin{array}{r}17 \\ 3\end{array}\right)=680$. Therefore the 68 blocks form a $3-(17,5,1)$ design.

Another way of showing that the 48 ovals form a $2-(16,5,4)$ co-design is to consider [ $\left.\begin{array}{lllll}1 & 4 & 7 & 10 & 13\end{array}\right],\left[\begin{array}{lllll}0 & 5 & 9 & 3 & 2\end{array}\right],\left[\begin{array}{lllll}13 & 4 & 14 & 6 & 8\end{array}\right]$, and $\left[\begin{array}{lllll}10 & 11 & 7 & 15 & 12\end{array}\right]$ as supplementary difference sets under the cycle $\mathrm{x} \rightarrow \mathrm{x}+1$ (mod 15$)$ with the symbol 0 fixed and 15 representing zero in the modular arithmetic. Then pairs containing the fixed element 0 each occur four times since there are four other elements in the set containing 0 . All differences 0 (mod 3) occur 4 times. Differences $\equiv 0 \quad(\bmod 3)$ each occur three times in the last three 
sets and five times in [ $\left.\begin{array}{llllll}1 & 4 & 7 & 10 & 13\end{array}\right]$; but this block is generated five times by the cycle $\mathrm{x} \rightarrow \mathrm{x}+1$ (mod 15) so differences from it should be weighted by a fifth. Thus all pairs not containing 0 occur four times. Hence, we have generated a $2-(16,5,4)$ design. Next compare stars, as before, to establish the correct triple count and so establish the $3-(17,5,1)$ design.

8. THE UNIQUENESS OF M(4).

Suppose B:[abcde] is a block in a co-design of AG(4). Then each pair of points from this block occurs once and once only in the blocks of $A G(4)$ which must therefore have ten blocks like $[\mathrm{ab} x y]$; one for each pair of points from $\mathrm{B}$, with $\mathrm{x}, \mathrm{y} \notin \mathrm{B}$. Two blocks of $\mathrm{AG}(4)$ intersect in at most one point so a particular $\mathrm{x}$ can occur at most twice in ten blocks of $A G(4)$ like [ab $x y]$ since $B$ can produce at most two disjoint pairs of points. On the other hand the block [ab $x y$ ] must intersect at least one of the blocks [cd...] and [ce...] of AG(4) for there cannot be two blocks on $c$ both parallel to [ $a b \mathrm{xy}]$. Therefore, each $\mathrm{x}$ occurs at least twice on the ten blocks like $[\mathrm{ab} x y]$. Since these blocks between them provide twenty $x, y$ spaces and each $x$ occurs just twice there are exactly ten values that $x$ (or $y$ ) can have. Therefore there is a point, 0 , which does not occur on any of the ten blocks like $\left[\begin{array}{ll}\mathrm{ab} & \mathrm{xy}\end{array}\right]$. Therefore the five blocks of $\mathrm{AG}(4)$ that intersect $B:[$ abcde] in just one point are patterned like

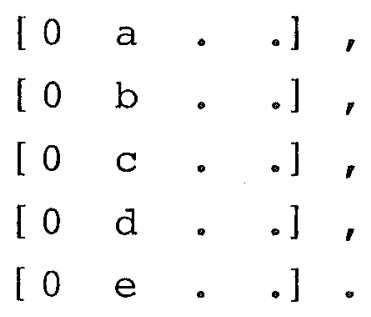

The point common to these five blocks, 0 , will be called the centre of $B$. Thus every block of the co-design has a uniquely defined centre and that centre does not lie on the block. In other words all the five lines from AG(4) which are tangent to a given oval (block of the co-design) are concurrent at the centre of the oval. The centre of an oval is determined once two tangents are known. Also, for any given oval from a point not on it there is either just one tangent line or there are five tangent lines. 


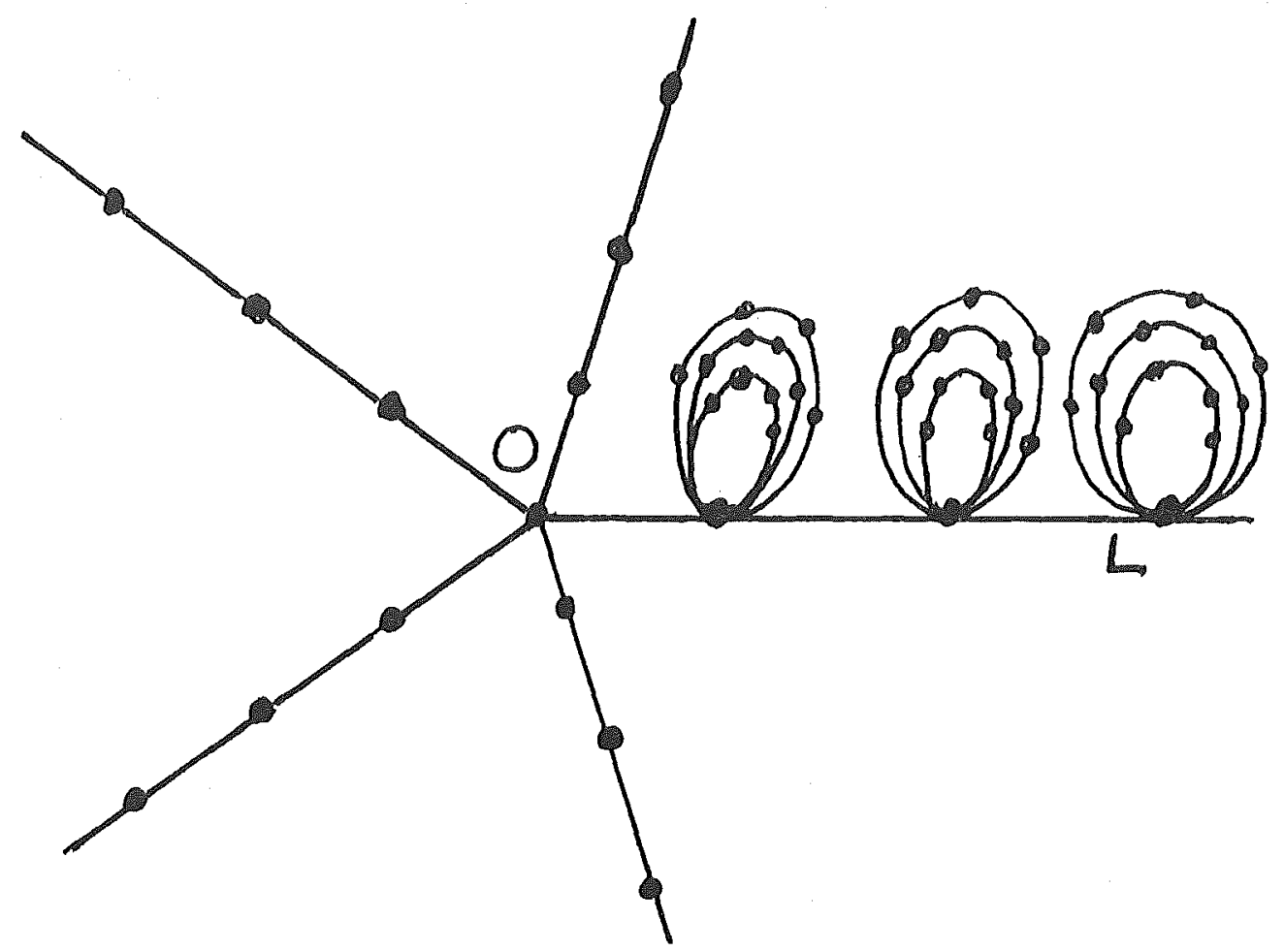

Figure 12. The configuration of the co-design ovals not through 0 but tangent to a given 1 ine $L$ through 0 , in $A G(4)$.

Warning: the points shown on the ovals, other than

the points of $\mathrm{L}$, appear more than once in the diagram
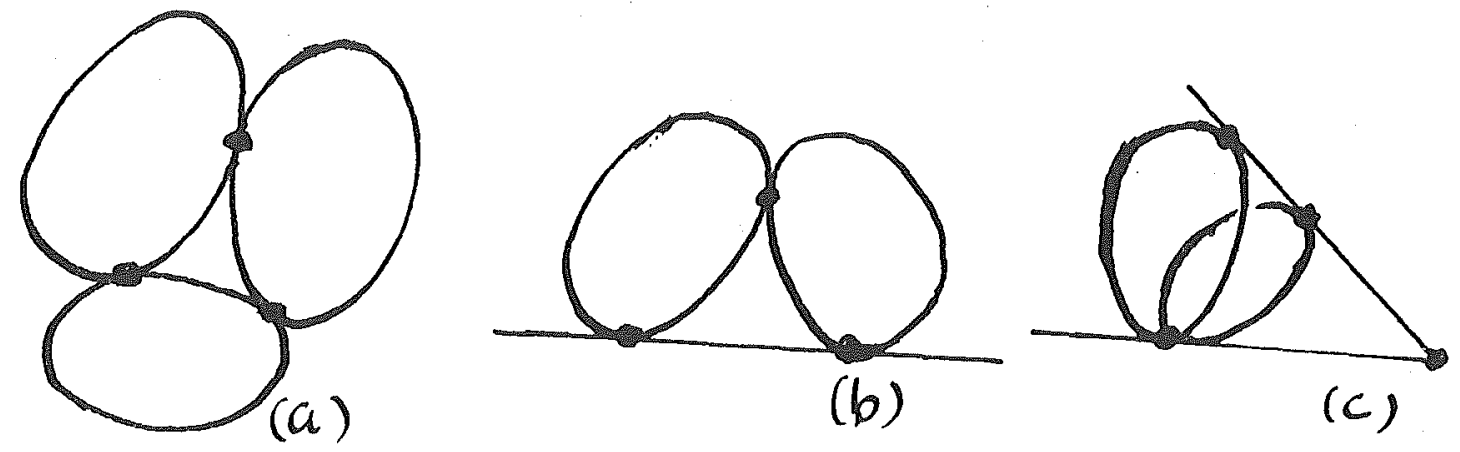

Figure 13. Impossible tangency configurations for ovals and lines in the extension of $\mathrm{AG}(4)$ to $M(4)$. 
Consider a line $L$ from $A G(4)$ and a point $P$ on it. Then through $\mathrm{P}$ there are 15 co-design ovals of which 12 cut the line again leaving three ovals all touching the line at $P$. No two of these ovals can have another point in common. If they did, since any three distinct non-collinear points lie on a unique oval, it would be impossible to account for the 12 ovals through $P$ which cut $\mathrm{L}$ elsewhere. Now consider the five lines through a fixed point 0 of $\mathrm{AG}(4)$, then omitting the tangencies at 0 , these five lines provide 45 line-oval tangencies, (figure 12). But there are 33 co-design ovals not through 0 . Of these let $\alpha$ be the number with a single tangent line from 0 and $\beta$ the number with 5 tangent lines from 0 . Then $\alpha+\beta=33$ and $\alpha+5 \beta=45$. From these $\beta=3$. Therefore each point of $A G(4)$ is the centre of exactly three ovals of the co-design.

If 3 co-design ovals touch two by two then they must do so at a single point for an extension by a new point $\infty$ to an $M(4)$ shows that the circles through a given point and tangent to a given circle all pass through a second point. Restrictions back down to $A G(4)$ 's on suitable points give the result for three ovals (and also for two ovals and a straight line touching two by two), (figure 13). Therefore each point of $A G(4)$ is the centre of exactly three mutually disjoint ovals, i.e. each point is the centre of a star whose ovals belong to the co-design.

All stars in AG(4) with the same centre are isomorphic the super-imposition of two suitably labelled star diagrams drawn as transparencies will provide a point permutation taking one to the other. Thus any star centred on the point 0 can be chosen. (There are in fact six possibilities). But once this choice has been made there is only one star centred on the point 1 which can be used and so the co-design is unique and consequently there is a unique $M(4)$.

9. THE IMPOSSIBILITY OF EXTENDING M(4) TO A 4-DESIGN.

For the current model of $A G(4)$ (section 7) the six stars centred on 0 in terms of their ovals are:- 
(a)

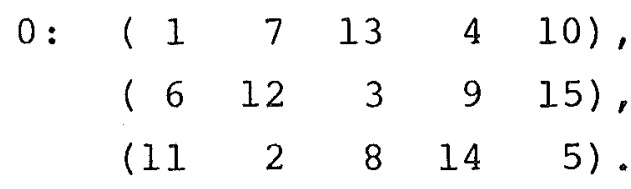

(c)

\begin{tabular}{|c|c|c|c|c|}
\hline $0:$ & (. 1 & 12 & 13 & 5 \\
\hline & $(6$ & 2 & 3 & 10 \\
\hline & (11 & 7 & 8 & 15 \\
\hline
\end{tabular}

(e) (b)

\begin{tabular}{|c|c|c|c|c|c|}
\hline $0:$ & $(1$ & 14 & 7 & 3 & 15) \\
\hline & $(6$ & 4 & 12 & 8 & 5) \\
\hline & (11 & 9 & 2 & 13 & 10) \\
\hline
\end{tabular}

(d)

$0: \quad \begin{array}{rrrrr}1 & 4 & 8 & 15 & 2), \\ (6 & 9 & 13 & 5 & 7), \\ (11 & 14 & 3 & 10 & 12) .\end{array}$

(f)

\begin{tabular}{|c|c|c|c|c|c|c|c|c|c|c|}
\hline $0: \quad(1$ & 10 & 12 & 8 & 9), & 0: & $(1$ & 5 & 3 & 9 & 2), \\
\hline$(6$ & 15 & 2 & 13 & 14), & & ( 6 & 10 & 8 & 14 & 7) \\
\hline (11 & 5 & 7 & 3 & 4). & & (11 & 15 & 13 & 4 & 12) \\
\hline
\end{tabular}

Two of these stars must be embedded in the 4-design. But the stars generated from using star (a) to make an $M(4)$ contain the ovals $\left(\begin{array}{lllll}0 & 5 & 9 & 3 & 2\end{array}\right),\left(\begin{array}{lllll}0 & 6 & 10 & 4 & 3\end{array}\right),\left(\begin{array}{lllll}0 & 7 & 11 & 5 & 4\end{array}\right),\left(\begin{array}{llll}0 & 12 & 6 & 5\end{array}\right)$,

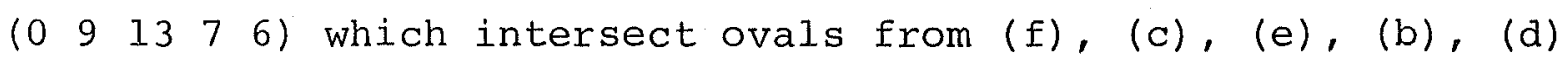
respectively in four points. Therefore, since in a $4-(18,6,1)$ design, every quartet of points must be on a unique block, the design cannot exist.

10. EXAMPLES OF $M(5), M(7)$ AND $M(8)$.

In this section are given examples of $M(5), M(7)$ and $M(8)$ with attendant star diagrams of $A G(5), A G(7)$ and $A G(8)$ all of which affine planes are unique. There is no $A G(6)$ since there is no projective plane of order 6 . No claims of uniqueness are made for the inversive planes. In fact two M(8)'s are known (e.g. see [3]). Examples of inversive planes of odd order are particularly interesting as questions about their characterisation are still unanswered. (i) $A G(5)$ and $M(5)$.

Figure 14 shows a star diagram for $A G(5)$ together with the line segments from two parallel classes. These classes may appear to be two different kinds but are in fact mathematically equivalent.

The PG(5) is generated cyclically from the starter block $\left[\begin{array}{llllll}1 & 5 & 11 & 24 & 25 & 27\end{array}\right]$ under the action of $\mathrm{x} \rightarrow \mathrm{x}+1 \bmod 31$. From the 31 blocks so generated delete the starter block and all points in 


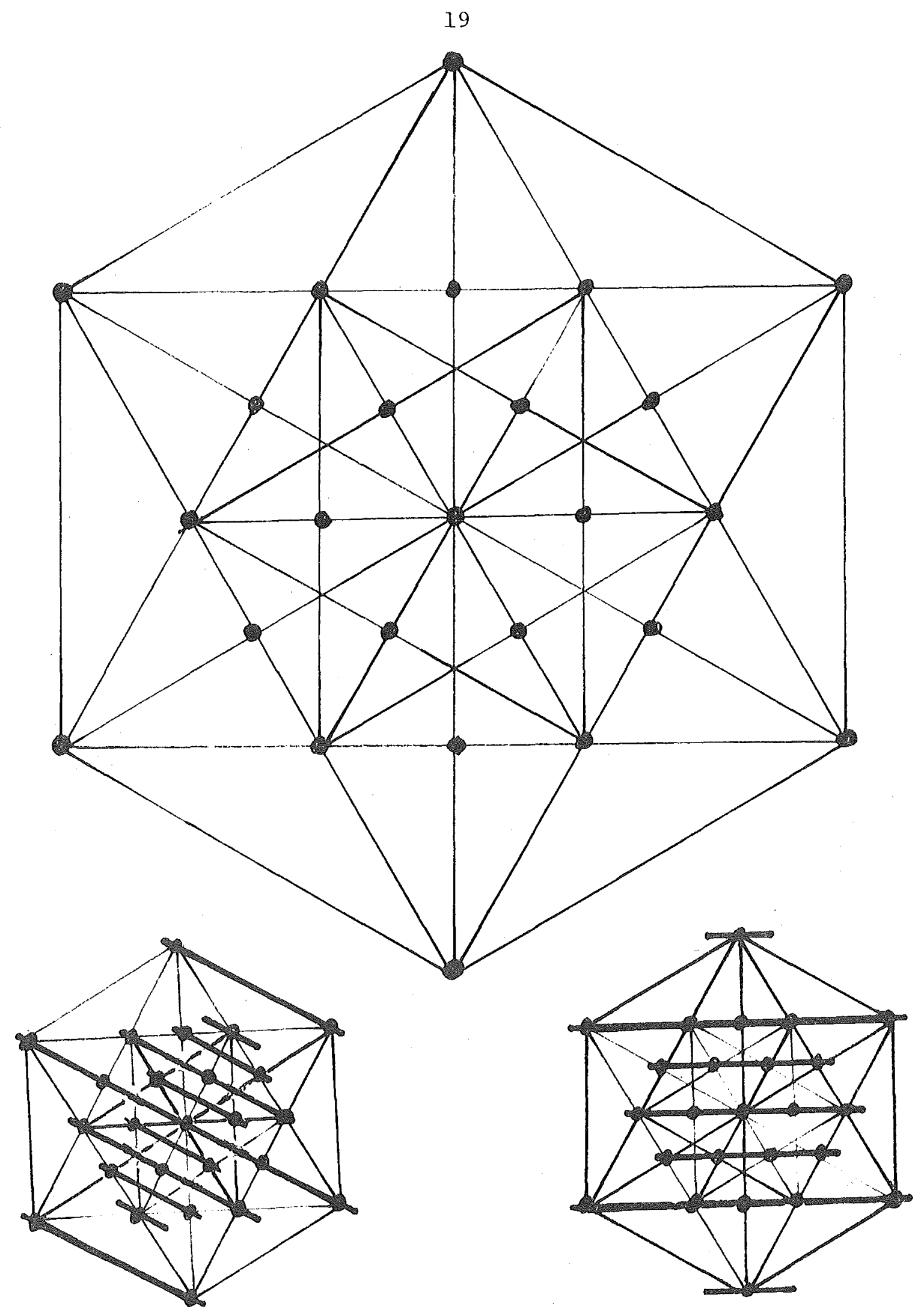

Figure 14. A star diagram for $A G(5)$ and line segments from two parallel classes. 


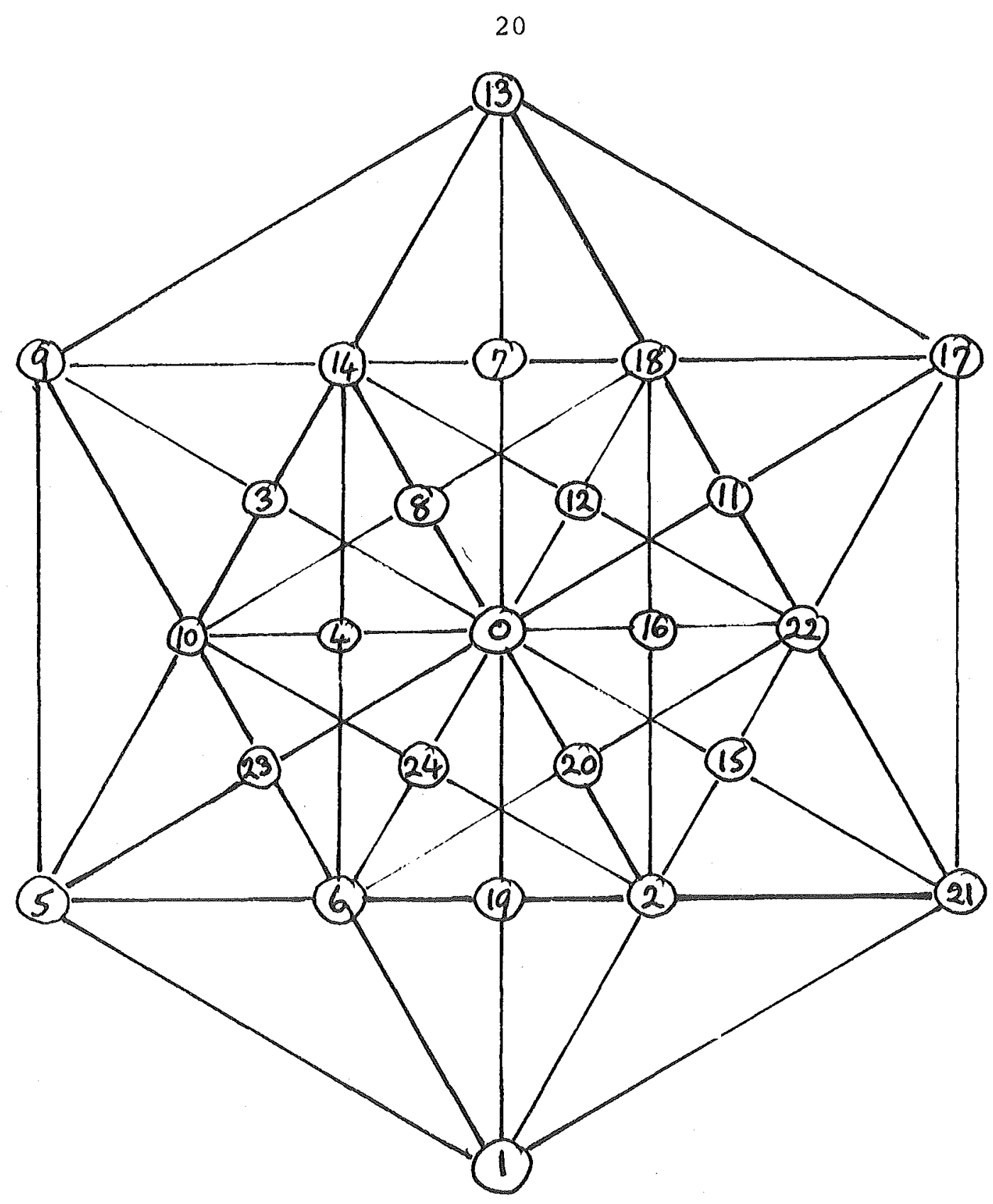

Figure 15. A labelled star diagram for $A G(5)$, Star A.

it to form a model of $A G(5)$. It is then convenient to take a new set of labels for the points:

$\begin{array}{rrrrrrrrrrrrrrrr}\text { Old labels: } & 0 & 2 & 3 & 4 & 6 & 7 & 8 & 9 & 10 & 12 & 13 & 14 & 15 & 16 & 17 \\ \text { New labels: } & 4 & 24 & 12 & 16 & 5 & 19 & 13 & 21 & 0 & 9 & 14 & 6 & 7 & 11 & 3 \\ & 18 & 19 & 20 & 21 & 22 & 23 & 26 & 28 & 29 & 30 & & & & \\ & 2 & 1 & 18 & 15 & 20 & 10 & 22 & 8 & 23 & 17 . & & & \end{array}$

Figure 15 is a labelled star centred on 0, Star A, corresponding to the $A G(5)$ with the new labels. 


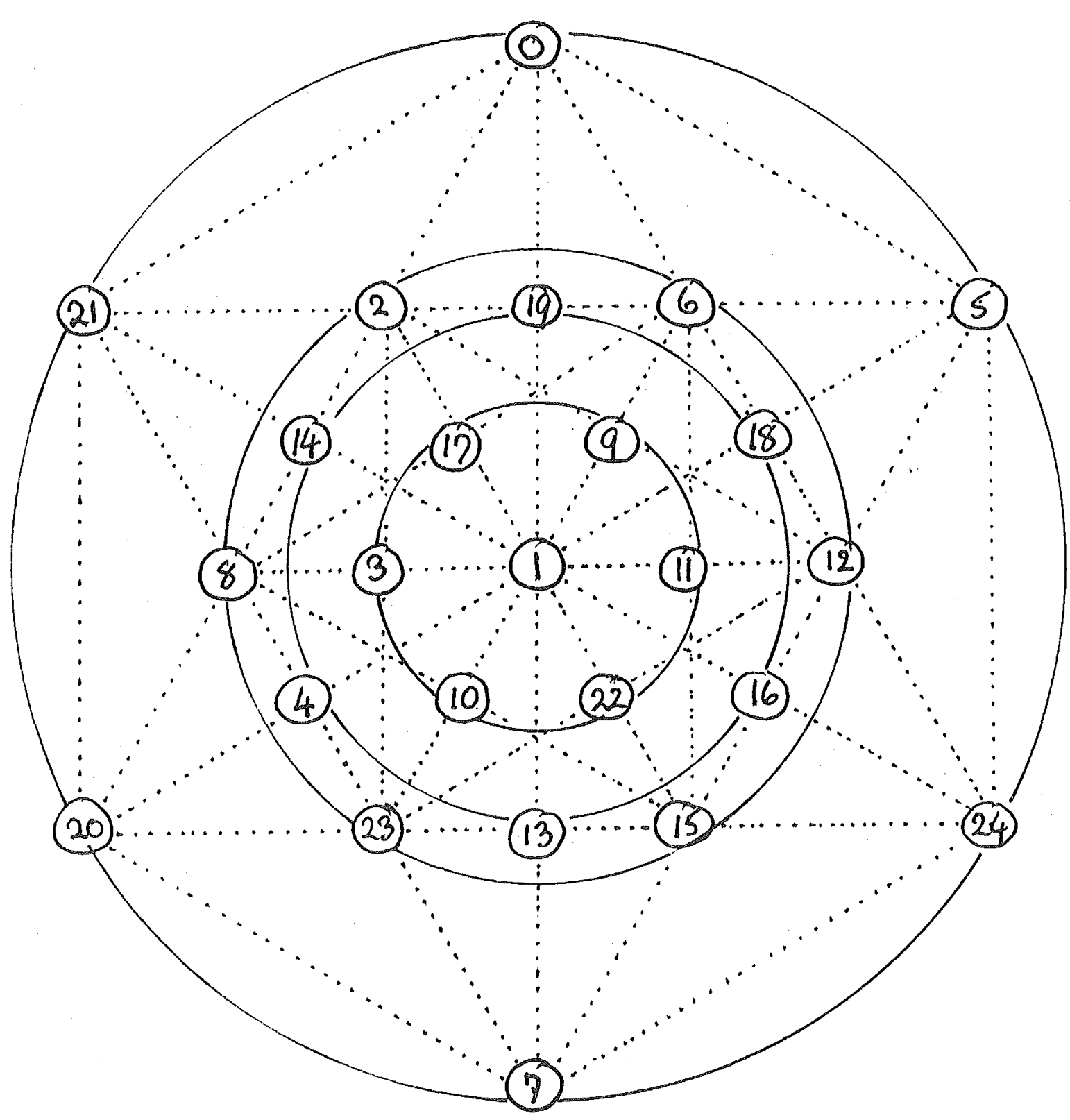

Figure 16. Another star for $A G(5)$, star B, displaying its ovals

Figure 16 gives another star, centred on 1, Star B, for the same $A G(5)$. The ovals are shown on the same diagram. The permutation (0) ( $\left.\begin{array}{lllll}1 & 2 & 3 & \ldots & 24\end{array}\right)$ preserves the ovals and centre of Star A. The point orbit induced by it on Star A is shown in. figure 17. Star B is not preserved by the above permutation which produces from it 24 stars in all. No oval from star $B$ intersects an oval from star $A$ in more than two points.

From here on the procedure follows that for constructing $M(4)$ and we have that 


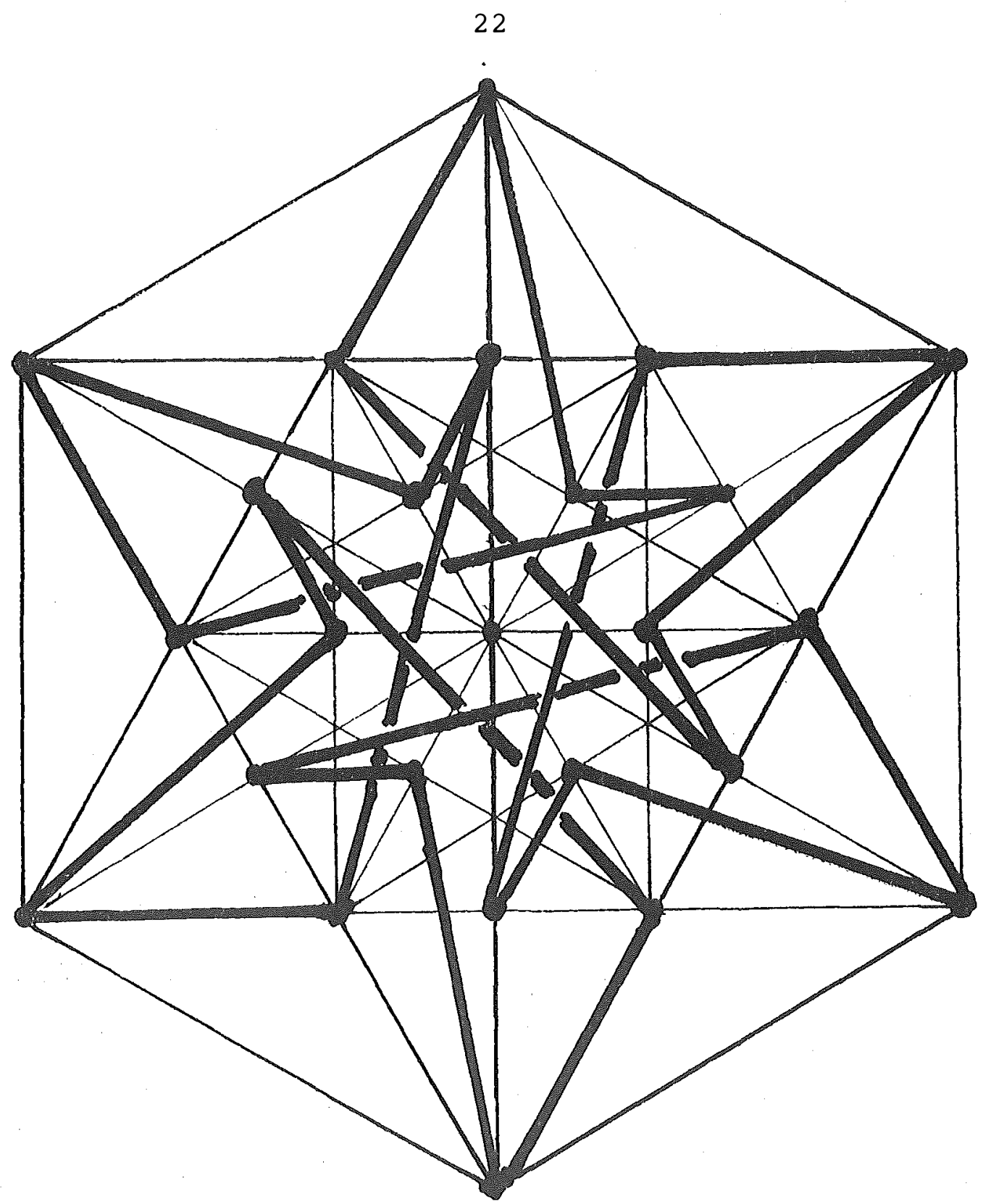

Figure 17. The point orbit induced on figure 15 by the permutation (0) $\left(\begin{array}{lllll}1 & 2 & 3 & \ldots & 24\end{array}\right)$.

the 130 blocks generated by the action of

$(\infty)(0)\left(\begin{array}{lllll}1 & 2 & 3 & \ldots & 24\end{array}\right)$ and $(\infty)(1)\left(\begin{array}{lllllllll}0 & 2 & 16 & 11 & 5 & 6 & 13 & 22 & 24\end{array}\right.$

$\begin{array}{lllllllllllllll}12 & 4 & 10 & 7 & 15 & 14 & 3 & 20 & 23 & 19 & 17 & 21 & 8 & 18 & 9 \text { ) on the blocks }\end{array}$

$\left[\begin{array}{llllll}\infty & 0 & 1 & 19 & 7 & 13\end{array}\right],\left[\begin{array}{llllll}1 & 5 & 9 & 13 & 17 & 21\end{array}\right]$,

$\left[\begin{array}{llllll}0 & 21 & 20 & 7 & 24 & 5\end{array}\right], \quad\left[\begin{array}{llllll}2 & 8 & 23 & 15 & 12 & 6\end{array}\right]$,

$\left[\begin{array}{llllll}19 & 14 & 4 & 13 & 16 & 18\end{array}\right],\left[\begin{array}{llllll}17 & 3 & 10 & 22 & 11 & 9\end{array}\right]$,

form a $3-(26,6,1)$ design i.e. an $M(5)$.

The last five blocks listed provide supplementary difference sets, with appropriate weighting for a $2-(25,6,5)$ co-design.

Here 0 is fixed and 24 is the zero element in the arithmetic modulo 24 . 
(ii) $A G(7)$ and $M(7)$.

The block [ $\left.\begin{array}{lllllllll}0 & 1 & 7 & 19 & 23 & 44 & 4 & 7 & 49\end{array}\right]$ is a cyclic difference set mod 57 which generates the 57 blocks of PG(7). If this block and the points on it are deleted to form an $A G(7)$ then it is convenient to relabel the points as follows with the old labels above and the new below:

$\begin{array}{rcccccccccccc}17 & 36 & 39 & 48 & 28 & 2 & 52 & 30 & 45 & 24 & 6 & 32 & 46 \\ 0 & 1 & 2 & 3 & 4 & 5 & 6 & 7 & 8 & 9 & 10 & 11 & 12 \\ & 10 & 51 & 22 & 56 & 9 & 3 & 26 & 14 & 29 & 38 & 20 & 5 \\ 13 & 14 & 15 & 16 & 17 & 18 & 19 & 20 & 21 & 22 & 23 & 24 \\ & 40 & 35 & 15 & 34 & 33 & 43 & 53 & 21 & 4 & 8 & 12 & 27 \\ 25 & 26 & 27 & 28 & 29 & 30 & 31 & 32 & 33 & 34 & 35 & 36 \\ 11 & 13 & 31 & 55 & 18 & 16 & 25 & 50 & 54 & 41 & 37 & 42 \\ 37 & 38 & 39 & 40 & 41 & 42 & 43 & 44 & 45 & 46 & 47 & 48 .\end{array}$

The new labels can then be entered onto star diagrams as in figures 18 and 19 to give Star A and Star B. No two ovals from these stars have more than two points in common. The families of parallel line segments are patterned after the fashion of those in the star diagrams for $\mathrm{AG}(5)$.

The 350 blocks of an $M(7)$ are provided by the action of $(\infty)(0)\left(\begin{array}{lllll}1 & 2 & 3 & \ldots & 48\end{array}\right)$ on the blocks

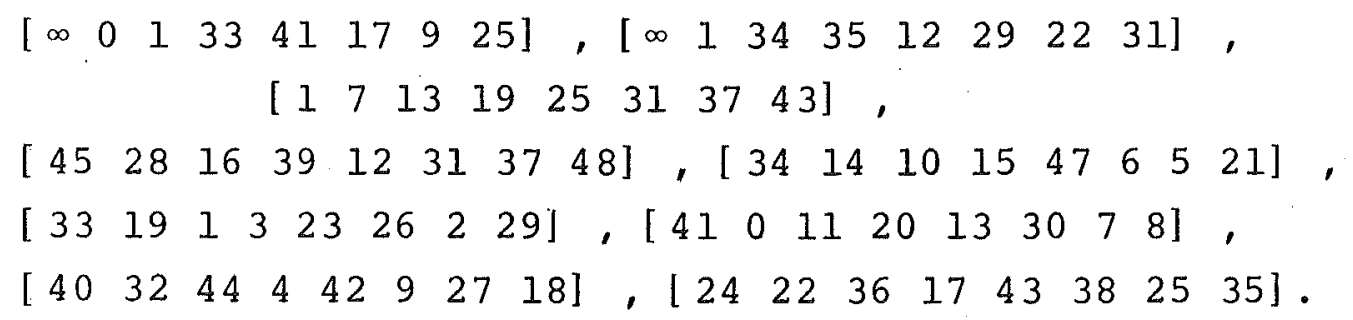

The last seven of the blocks listed provide supplementary difference sets for a $2-(49,8,7)$ co-design. 
(iii) AG(8) and an M(8).

The block [ $\left.1 \begin{array}{lllllllll}1 & 2 & 4 & 8 & 16 & 32 & 64 & 55 & 37\end{array}\right]$, consisting of all powers of $2(\bmod 73)$, is a cyclic difference set for PG(8). Deletion of this block and all points on it yields an $A G(8)$. In this case the points retained will not be relabelled and the deleted points will be used as labels for the appropriate parallel classes.

A star diagram for $A G(8)$ is given in figure 20 together with a family of parallel line segments and a diagram of the ovals. To construct Star A choose a line containing an outside vertex and the centre of the star diagram. Then label the points in order along this line from the vertex through the centre with $7,31,3,0,63$, 36, 15, 54, so 0 falls on the centre of the star. Then starting

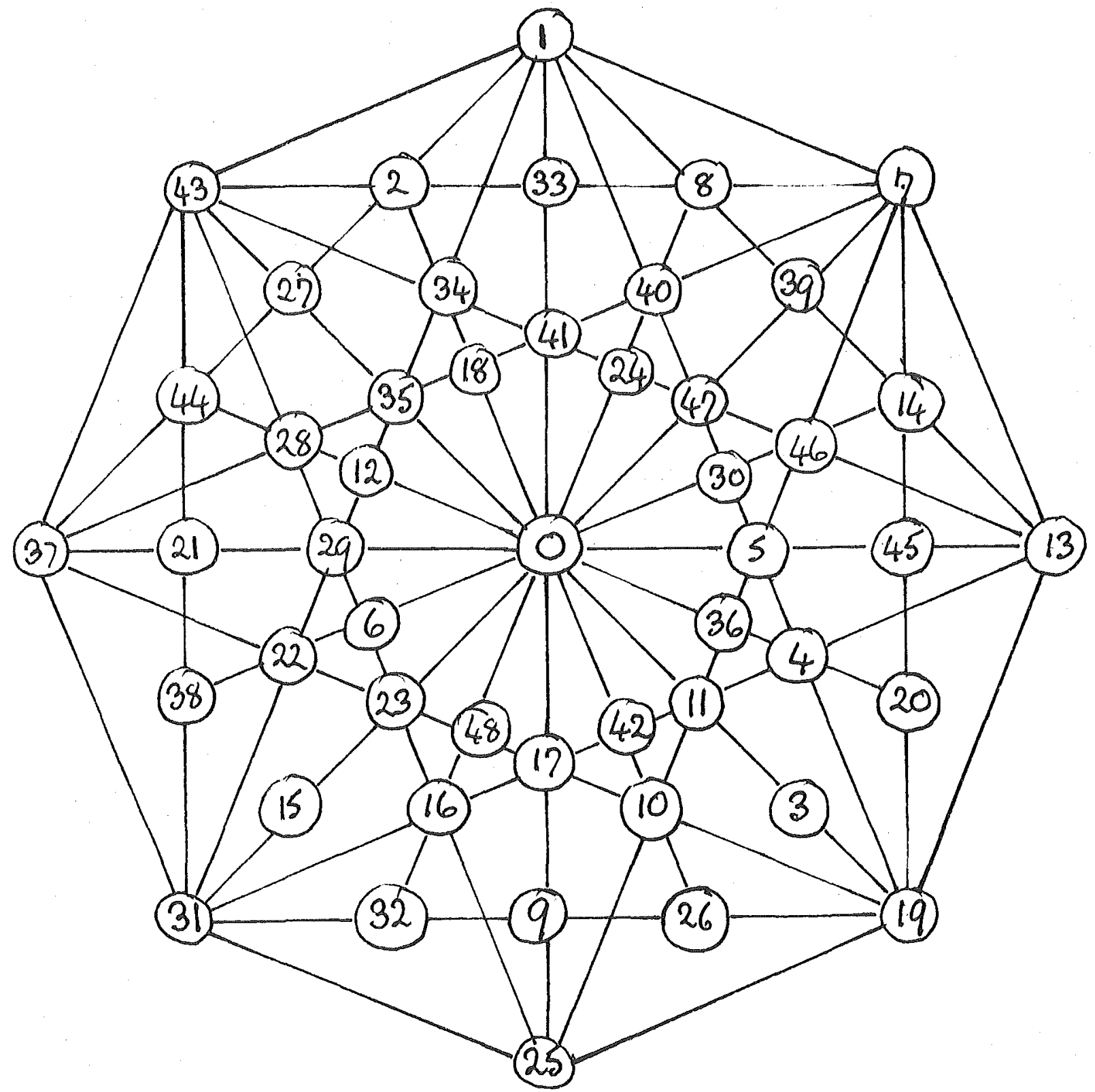

Figure 18. Star A for AG(7). 


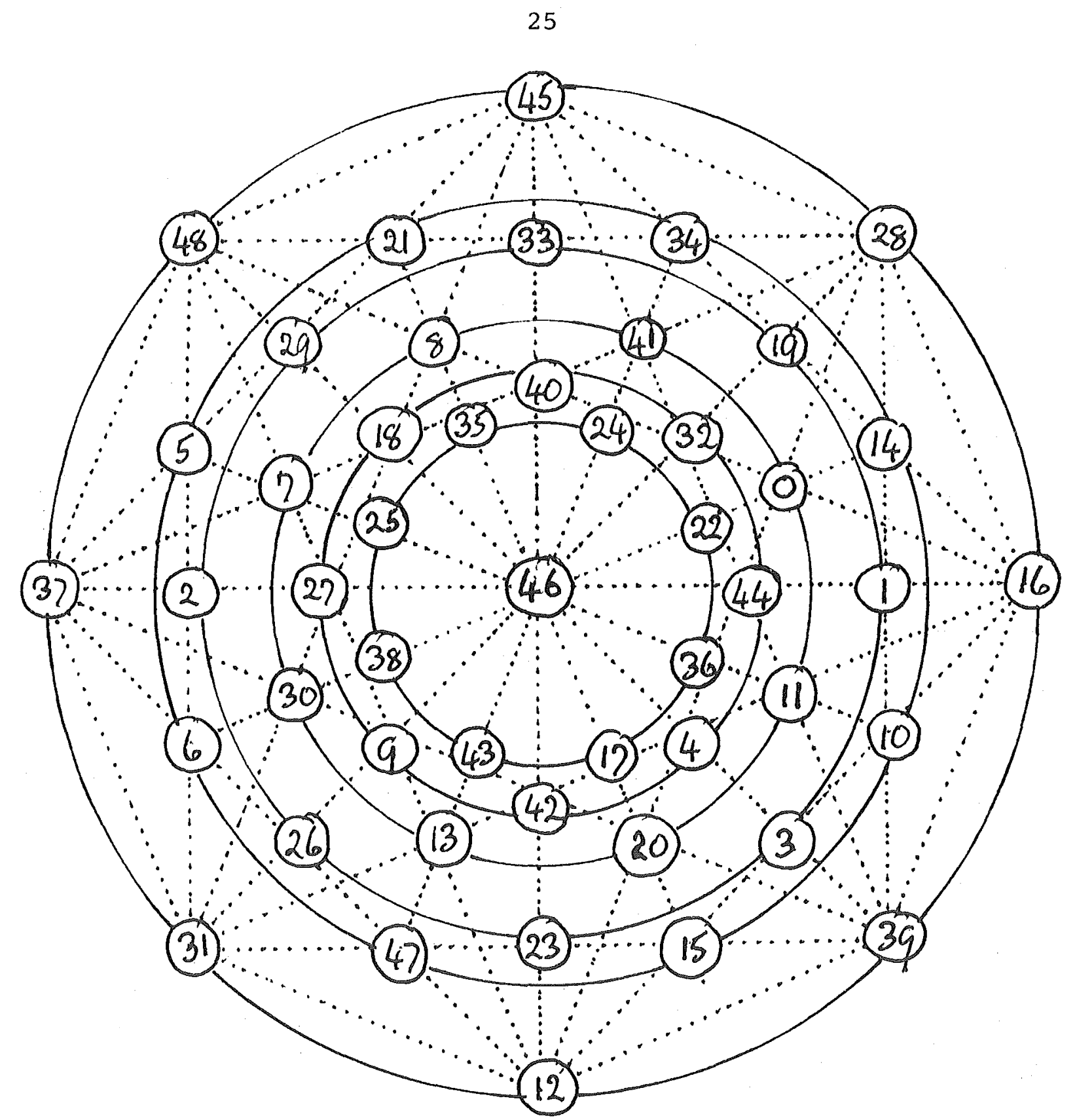

Figure 19. Star B and its ovals for AG(7).

with the outermost oval and working inwards the ovals, as ordered sets of points, can be entered from the table:

Ovals in Star A

\begin{tabular}{l|rrrrrrrrr} 
Oval & \multicolumn{10}{|c}{} \\
\hline 1 & $(12$ & 68 & 58 & 43 & 9 & 47 & 62 & 7 & $17)$ \\
2 & $(29$ & 53 & 54 & 41 & 71 & 44 & 59 & 49 & $50)$ \\
3 & $(66$ & 30 & 15 & 25 & 60 & 52 & 21 & 23 & $34)$ \\
4 & $(27$ & 48 & 57 & 20 & 69 & 6 & 31 & 46 & $28)$ \\
5 & $(24$ & 35 & 36 & 10 & 33 & 18 & 61 & 5 & $26)$ \\
6 & $(51$ & 40 & 39 & 45 & 22 & 56 & 14 & 3 & $11)$ \\
7 & $(67$ & 72 & 63 & 13 & 70 & 38 & 65 & 42 & $19)$
\end{tabular}



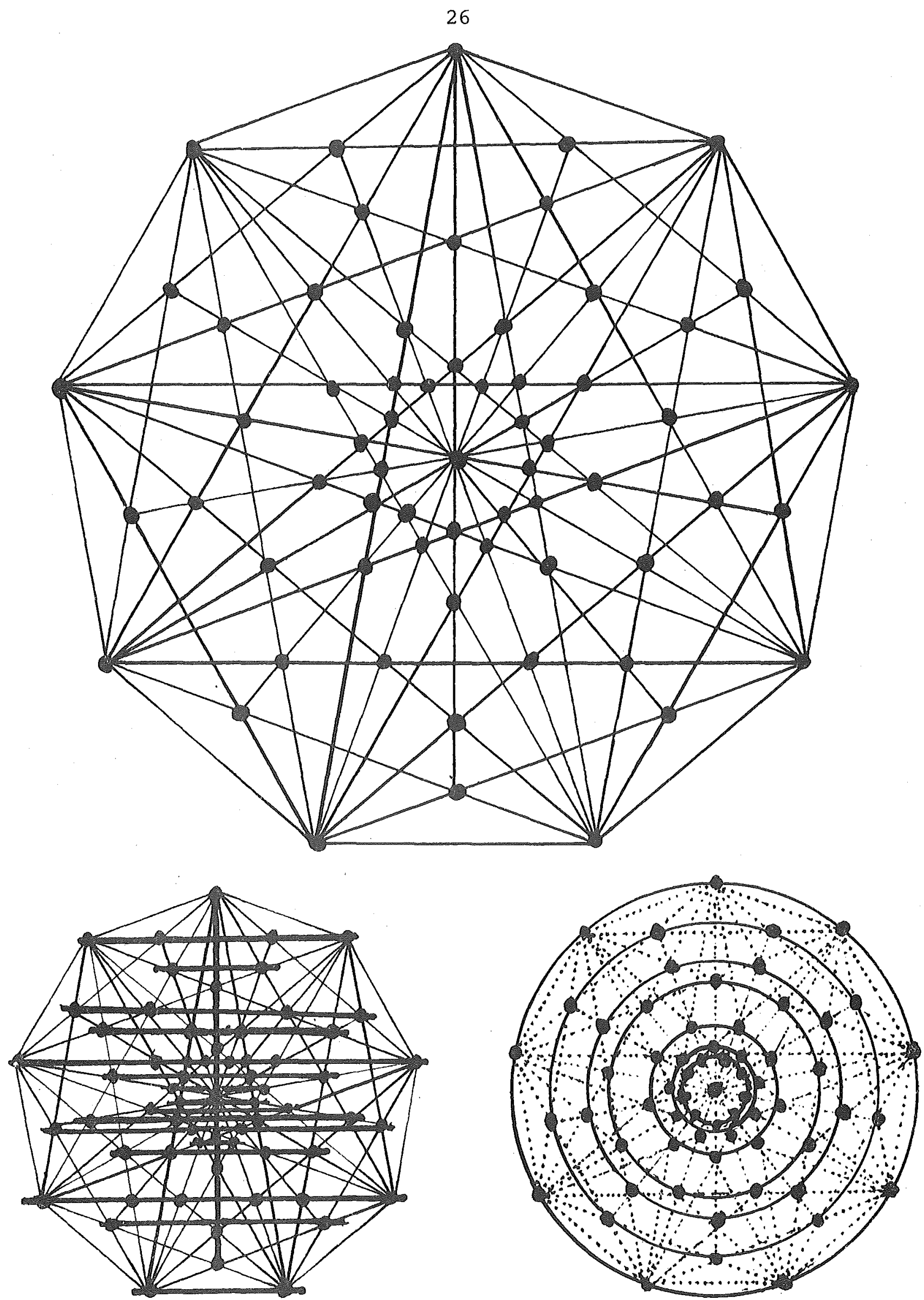

Figure 20. A star diagram for $A G(8)$ together with a family of parallel line segments and its seven ovals. As usual, not all the line segments are drawn in. 
Through the centre 0 of Star $A$ are the

Radial Lines in Star A

\begin{tabular}{|c|c|c|c|c|c|c|c|c|}
\hline Parallel Class & Centre & \multicolumn{7}{|c|}{ Other Points } \\
\hline $1 y$ & 0 & $\pi^{3}$ & $h^{36}$ & $a^{1}$ & $d^{54}$ & 63 & 15 & 17 \\
\hline $32 \downarrow$ & 0 & 742 & $T_{23}$ & $T_{43}$ & $T_{45}$ & \$ 5 & $T_{57}$ & 49 \\
\hline 64 & 0 & 10 & 46 & 41 & 13 & 25 & 17 & 11 \\
\hline 55 & 0 & 34 & 9 & 22 & 26 & 20 & 50 & 19 \\
\hline 4 & 0 & 28 & 71 & 70 & 60 & 12 & 51 & 33 \\
\hline 8 & 0 & 47 & 56 & 24 & 69 & 29 & 67 & 66 \\
\hline 37 & 0 & 44 & 38 & 52 & 68 & 40 & 18 & 27 \\
\hline 2 & 0 & $14 \uparrow$ & 351 & $\sigma_{1}$ & $53 \hat{~}$ & $72 \mathrm{~A}$ & 301 & 62 \\
\hline 16 & 0 & 65 & 21 & 5 \& & 39) & 61 & 48 & 59 \\
\hline & (fixed) & & & un & rph & $m \alpha)$ & & \\
\hline
\end{tabular}

This table also gives an automorphism of $A G(8)$ which fixes star $A$ by taking ovals onto ovals and fixing the centre. The point permutation for this automorphism, $\alpha$, is given by reading down successive columns from left to right in the "other points" section. As indicated the parallel classes are cycled at the same time.

To construct Star B (with centre 3) take as points in order along a radial line, $31,7,0,3,15,54,63,36$. Then enter up the

\section{Ovals in Star B}

\begin{tabular}{l|rrrrrrrrr} 
Oval & \multicolumn{10}{|c}{} \\
\hline 1 & 18 & 25 & 44 & 50 & 30 & 26 & 17 & 31 & 62 \\
2 & 52 & 38 & 36 & 19 & 34 & 58 & 49 & 59 & 43 \\
3 & 60 & 9 & 63 & 68 & 66 & 29 & 20 & 27 & 71 \\
4 & 39 & 23 & 67 & 70 & 21 & 45 & 33 & 7 & 24 \\
5 & 46 & 56 & 54 & 51 & 6 & 12 & 13 & 72 & 47 \\
6 & 10 & 22 & 28 & 69 & 40 & 35 & 65 & 0 & 42 \\
7 & 48 & 5 & 15 & 61 & 57 & 53 & 14 & 11 & 41
\end{tabular}


Thus through the centre, 3, we have the

Radial Lines of Star B

\begin{tabular}{c|c|ccccccc} 
Parallel Class & Centre & \multicolumn{7}{|c}{ Other Points } \\
32 & 3 & Puy $_{11}^{0}$ & 54 & 7 & 36 & 15 & 63 & 31 \\
64 & 3 & 27 & 50 & 69 & 72 & 70 & 59 \\
55 & 3 & 51 & 24 & 19 & 61 & 68 & 62 & 42 \\
4 & 3 & 71 & 30 & 40 & 47 & 21 & 43 & 41 \\
8 & 3 & 39 & 34 & 57 & 66 & 18 & 10 & 6 \\
37 & 3 & 26 & 35 & 46 & 45 & 52 & 48 & 60 \\
2 & 3 & 58 & 53 & 29 & 25 & 46 & 12 & 23 \\
16 & 3 & 65 & 56 & $33 \uparrow$ & 38 & $5 \uparrow$ & 9 & 17 \\
& 3 & 14 & 20 & 44 & 28 & 13 & 67 & 49
\end{tabular}

The application of automorphisms $\alpha$ and $\beta$ to Star A and Star B will produce 64 stars yielding 448 ovals. Since no two ovals from Star A and Star B have more than two points in common, by the double transitivity on the stars induced by $\alpha$ and $\beta$, a co-design for $A G(8)$ is produced. Thus an $M(8)$ is constructed.

\section{I1. CONSTRUCTING A CLASSIC 5-DESIGN}

... when Lord Carnarvon, unable to stand the suspense any longer, inquired anxiously, "Can you see anything?" it was all I could do to get out the words, "Yes, wonderful things." Then, widening the hole a little further, so that we both could see, we inserted an electric torch.

Howard Carter, The Tomb of Tutankhamen.

Figure 21 illustrates the machinery of ovals and lines in $A G(3)$. This geometry, which is unique, has 9 points and 12 lines. Figure 21 (ii) illustrates a star preserving automorphism by way of a point orbit fixing the centre of the star.

Let the points of $A G(3)$ be labelled $0,1,2, \ldots, 8$. Then blocks (lines) in the corresponding $2-(9,3,1)$ design in their parallel classes can be taken as

$$
\begin{aligned}
& {\left[\begin{array}{lll}
1 & 8 & 3
\end{array}\right],\left[\begin{array}{lll}
1 & 6 & 7
\end{array}\right],\left[\begin{array}{lll}
8 & 2 & 7
\end{array}\right],\left[\begin{array}{lll}
5 & 6 & 8
\end{array}\right] \text {, }} \\
& {\left[\begin{array}{lll}
6 & 0 & 2
\end{array}\right],\left[\begin{array}{lll}
8 & 0 & 4
\end{array}\right],\left[\begin{array}{lll}
1 & 0 & 5
\end{array}\right],\left[\begin{array}{lll}
7 & 0 & 3
\end{array}\right] \text {, }} \\
& {\left[\begin{array}{lll}
7 & 4 & 5
\end{array}\right],\left[\begin{array}{lll}
3 & 2 & 5
\end{array}\right],\left[\begin{array}{lll}
3 & 6 & 4
\end{array}\right],\left[\begin{array}{lll}
4 & 2 & 1
\end{array}\right] \text {. }}
\end{aligned}
$$




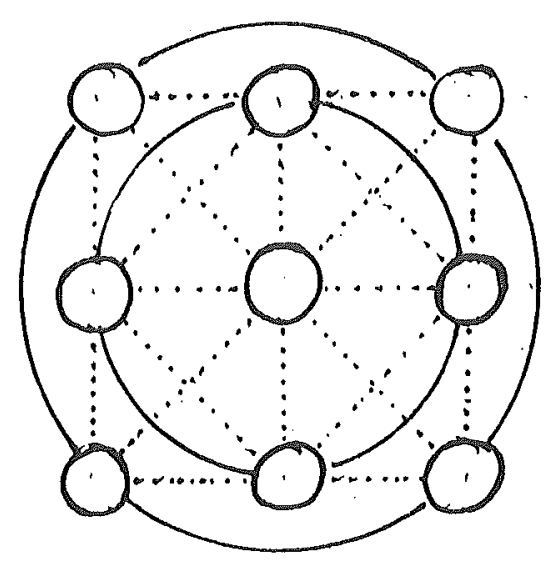

(i)

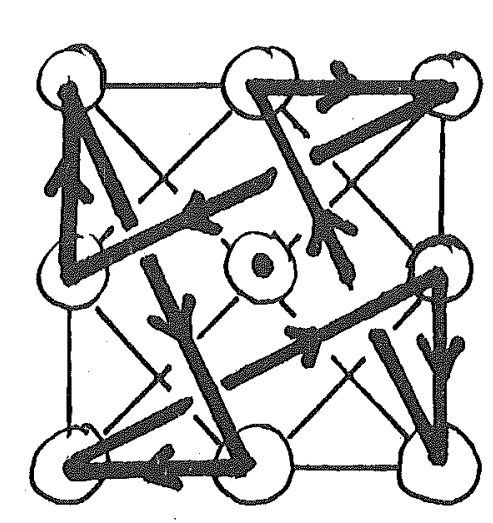

(ii)

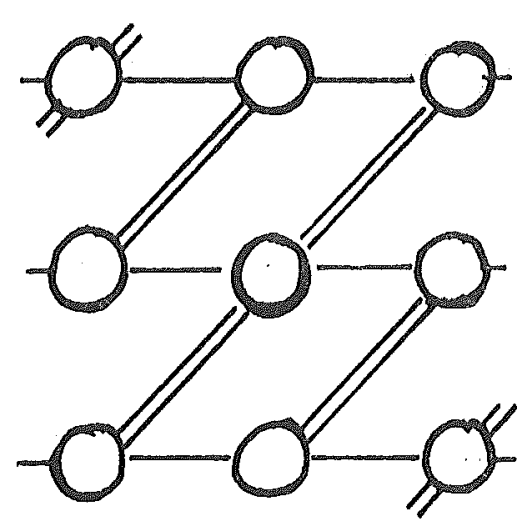

(iii)

Figure 21. (i) Ovals and lines in $\mathrm{AG}(3)$.

(ii) A star preserving orbit for AG(3).

(iii) Two families of parallel segments.

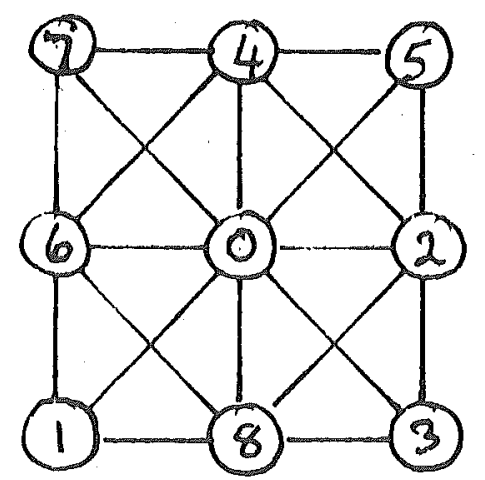

(a)

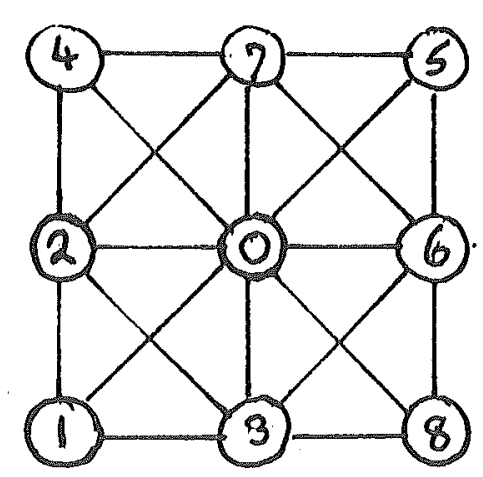

(b)

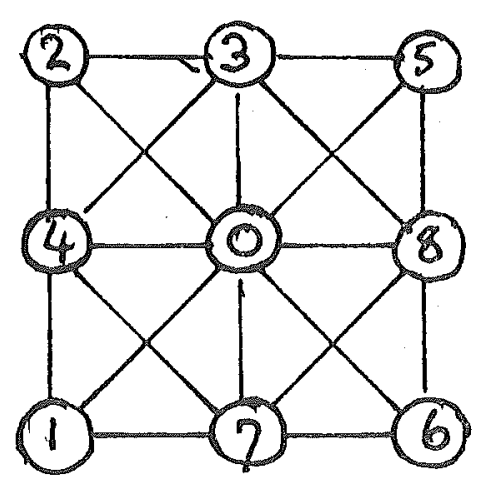

(c)

Figure 22. The three 0-centred stars of $A G(3)$.

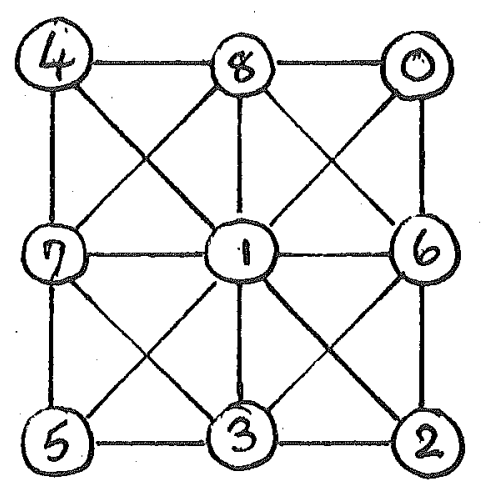

(a)

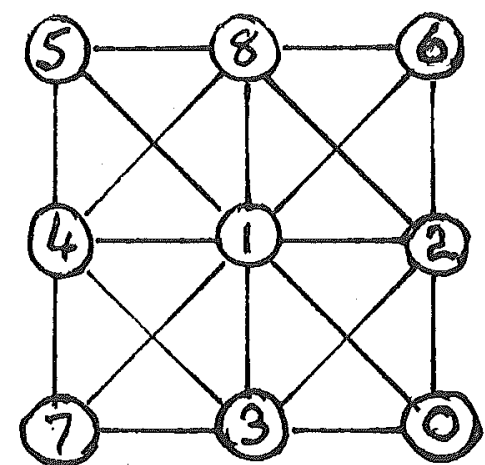

(b)

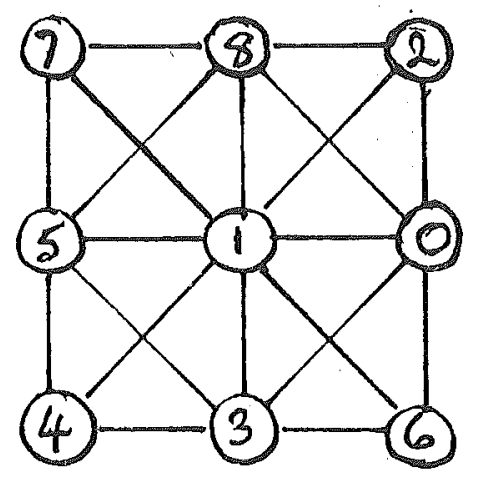

(c)

Figure 23. The three 1-centred stars of $A G(3)$. 
The three possible 0-centred stars corresponding to these blocks are given in figure 22. The labels a, b, $c$ there used will also do duty as extension points. In figure 23 the three 1-centred stars are shown. Each of these is directly below the only o-centred star neither of whose ovals cut either oval from it in more than two points.

The automorphism (0) (l $\left.\begin{array}{llllllll}1 & 2 & 3 & 4 & 5 & 6 & 7 & 8\end{array}\right)$ applied to figure 22 (a) and figure 23 (a) produces 9 stars and 18 ovals that make a 2 - $(9,4,3)$ co-design for the $\operatorname{AG}(3)$. A $3-(10,4,1)$ design, an $M(3)$, is then produced by adding the extension point a to each line of the geometry. This process works just as well with figures 22 (b) and 23 (b) or figures 22 (c) and 23 (c) with automorphisms provided by the orbit diagram of figure 21 (ii). In other words the $2-(9,3,1)$ has three $2-(9,4,3)$ co-designs and no more. These three co-designs are however isomorphic. Indeed, of the three permutations,

$\alpha:(0)(a)(b c)\left(\begin{array}{llllllll}1 & 2 & 3 & 4 & 5 & 6 & 7 & 8\end{array}\right)$,

$\beta:(0)(b)(c a)\left(\begin{array}{llllllll}1 & 6 & 8 & 7 & 5 & 2 & 4 & 3\end{array}\right)$,

$\gamma:(0)(c)(a b)\left(\begin{array}{llllllll}1 & 8 & 6 & 3 & 5 & 4 & 2 & 7\end{array}\right)$,

any one fixes one star of figure 22 and interchanges the other two together with their labels a, b, c. It also fixes one column of stars generated from figure 23 and permutes the stars of the other two columns together with the labels $a, b, c$.

Now to extend to a $4-(11,5,1)$ design, blocks of five points 110 four of which lie on a circle of $M(3)$ are required. These are formed by taking centred ovals from stars; that is, to each oval add the centre of the star to which it belongs. There are as many centred ovals as ovals. Then from figure 22 take any two of the stars; add to the ovals generated by each (with the help of figure 23) the label of the other; add both labels to each line of $A G(3)$; and throw in the centred ovals generated by the third star of figure 22. The resulting 66 blocks of five points each, taken from a supply of 11 points, form a $4-(11,5,1)$ design.

Yet a further, and final, extension is possible, yielding a 5 - $(12,6,1)$ design. Take the three stars of figure 22; add the label of each to the centred ovals generated by it; to the 
ovals generated by each of the stars the labels of the other two; add all three labels to the lines of $A G(3)$. By now we have 120 blocks of 6 points drawn from a supply of 12 points. But a 5 - $(12,6,1)$ design has 132 blocks. The further 12 blocks needed to complete the design come from taking the complements with respect to $\{0,1,2,3,4,5,6,7,8\}$ of the lines of $\mathrm{AG}(3)$ - could such blocks be called comp-lines?* They are made by taking the union of any two lines from the same parallel class. In fact a 5 - $(12,6,1)$ design has to be self-complementary so the choice of blocks in extending from the 4-design to the 5-design is forced upon us.

\begin{tabular}{|c|c|c|}
\hline \multicolumn{2}{|c|}{$\begin{array}{c}\text { Lines of AG(3)+a b c } \\
12 \text { blocks }\end{array}$} & Ovals $+\mathrm{ab}$ \\
\hline Ovals $+\mathrm{bc}$ & Ovals $+\mathrm{ca}$ & 18 blocks \\
\hline 18 blocks & 18 blocks & 18 blocks \\
\hline 18 blocks & Centred ovals $+\mathrm{b}$ & Centred ovals $+\mathrm{c}$ \\
\hline & 18 blocks & Line complements of $\mathrm{AG}(3)$ \\
\hline
\end{tabular}

Figure 24. Scheme for constructing the 132 blocks of a $5-(12,6,1)$ design.

Of course it has not been proved that we do indeed have a 5-design. This can be done by careful counting of quartets and quintuples of points. However let us take advantage of the knowledge of some of the elements of the automorphism group. We already have three such elements $\alpha, \beta, \gamma$. Now let us undo the construction by taking restrictions on symbols other than $a, b, c$; for example on the triple $0,1,2$. Then the AG(3) reappears, with a different labelling, as the blocks

* cf: 'Please don't ex-pline, show me.' : Eliza Doolittle 


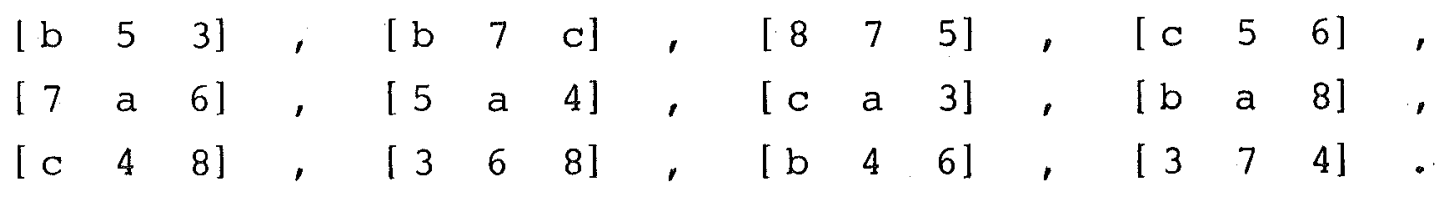

Then a return to figures $21,22,23$ suitably relabelled allows more automorphisms of the putative 5-design to be unearthed. Thus use of a-centred stars and $0,1,2$ as the extension points gives the automorphisms $\delta, \varepsilon, \zeta$ listed below together with $\alpha, \beta, \gamma$. A seventh automorphism $\eta$ taken from figure 21 (ii) applied to the 1-centred star of figure 23 (a) is added.

$$
\begin{aligned}
& \alpha:(0)(a)(b c)\left(\begin{array}{llllllll}
1 & 2 & 3 & 4 & 5 & 6 & 7 & 8
\end{array}\right) \text {, } \\
& \beta:(0)(b)(c a)\left(\begin{array}{llllllll}
1 & 6 & 8 & 7 & 5 & 2 & 4 & 3
\end{array}\right) \text {, } \\
& \gamma:(0)(c)(a b)\left(\begin{array}{llllllll}
1 & 8 & 6 & 3 & 5 & 4 & 2 & 7
\end{array}\right) \text {, } \\
& \delta:(a)(1)(20)\left(b \begin{array}{lllllll}
6 & 3 & 4 & 8 & 7 & c & 5
\end{array}\right) \text {, }
\end{aligned}
$$

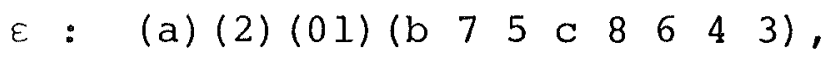

$$
\begin{aligned}
& \zeta:(a)(0)(12)\left(b \begin{array}{lllllll}
5 & 7 & 3 & 8 & 4 & 6 & c
\end{array}\right) \text {, } \\
& \eta:(1)(a)(b c)\left(\begin{array}{llllllll}
2 & 8 & 0 & 7 & 4 & 3 & 5 & 6
\end{array}\right) \text {. }
\end{aligned}
$$

These permutations between them are transitive on the 12 symbols which implies transitivity of the automorphism group, $G$, of the 132 aforesaid blocks. The sub-group of $G$ fixing 0 say contains $\alpha, \beta, \gamma, \zeta$, implying 2-transitivity for $G$. The subgroup of $G$ fixing 0 and a contains $\alpha$ and $\zeta$, implying 3-transitivity for $G$. The subgroup of $G$ fixing $a, b, c$ contains $\alpha^{2}, \beta^{2}, \gamma^{2}$ and $n^{2}$, so $G$ is 4-transitive. The sub-group of $G$ fixing $0, a, b, c$ contains $\alpha^{2}, \beta^{2}, \gamma^{2}$ so $G$ is 5-transitive. Thus the 132 blocks of points on which $\mathrm{G}$ acts form a 5-design.

It is well known that $G$ is $M_{12}$, the Mathieu group of order 12.11.10.9.8. (Witt [ 13]), one of the sporadic simple groups that both delight and irk group theorists.

\section{DEMBOWSKI'S THEOREM}

To explain this theorem an excursion into three dimensional finite space is necessary. A point set, $S$, in such a space is called an ovoid if (i) any straight line of the space meets $S$ in at most two points and if (ii) for any point $P$ of $S$ the lines through $P$ and no other point of $S$ contain all the points of a plane. (Think of an ellipsoid in Euclidean 3-space with its secant and 
tangent lines). The non-trivial plane sections of an ovoid are the circles of an inversive plane. It is not known if there are inversive planes which cannot be derived from an ovoid. If there are any such then they must be of odd order by the theorem of Dembowski [ 3 ] that

Every inversive plane of even order $n$ is isomorphic to the system of points and plane sections of an ovoid in a threedimensional projective space over the Galois field GF(n).

Since Galois fields of even order $\mathrm{n}$ exist only when $\mathrm{n}$ is a power of 2 it follows that extendible finite affine planes can exist only under the same conditions. A second consequence of Dembowski's Theorem is that in any inverse plane of even order Desargues' theorem must hold.

In proving his theorem Dembowski used a property of finite planes of even order, namely, all the tangents to an oval have a point in common. He showed that this implies that any three circles touching two by two have a point in common (and here let me admit that the methods used in section 8 owe much to this result). He then proved that all the circles, tangent to two disjoint circles pass through two uniquely determined points $P$ and $Q$.

Now if there are $n-1$ mutually disjoint circles in a finite inversive plane then the $P$ and $Q$ must be the same for all of them. Then by labelling $P$ and $Q$ with 0 and $\infty$ followed by a restriction on $\infty$ we have a star centred on 0 in the resulting finite affine plane. One can imagine that the star diagrams of earlier sections are shadows of a wire model which has been turned so that $\infty$ is hidden behind 0 the centre of the star. Any inversive plane of even order $n$ must be starlike and each point must be the centre of an affine star; for, following the ideas of section 8, in a finite affine plane which is a restriction of an inversive plane of order n $(=2 m)$ there are $n^{3}-2 n^{2}+1$ ovals which are also circles in the inversive plane and which do not pass through a given point $P$. Each of the $n+1$ lines through $P$ contains $n-l$ points other than $P$ and there are $(n-1)$ line/oval tangencies at each. From $P$ there is either one tangent or $(n+1)$ tangents to an oval not through $P$. Let $\alpha$ be the number of ovals with a single tangent, 
and $\beta$ the number with multiple tangents, from $P$. Then by counting line/oval tangencies we have

$$
\alpha+(n+1) \beta=(n-1)^{2}(n+1)
$$

and $\alpha+\beta=n^{3}-2 n^{2}+1 ;$

so $\beta=n-1$. Then Dembowski's subtle result on the tangencies of three circles assures us that these $\beta$ ovals must be mutually disjoint.

Must finite affine planes of odd order be starlike if they are to be extended to inversive planes? If the answer is yes then the ovals in a star must all be associated with a point fit to be called the centre of each. The difficulty then lies in identifying this point being given only one oval from a star. The tangent lines to an oval in a finite plane of odd order do not have a point in common so the mechanism used for the finite planes of even order is not available. However, in a finite plane of odd order on a line $I$ not intersecting a given oval 0 the points fall into two sets of equal size. From a given point on $L$ there are either just two tangents to o or there are none at all. If $L$ is taken to be the line at infinity in forming an affine plane then the tangent lines from points on it to o form pairs of parallel lines. The lines joining the two points of tangency on o defined by each parallel pair are secants of $O$. If these secants have a common point then this surely must be the centre of the star to which 0 belongs, supposing such a star exists. The star diagrams presented for $A G(5), A G(7)$ and even $A G(3)$ support this notion. However, such visual evidence may be misleading since the geometries concerned are all Desarguesian and have nice transitivity and cyclic properties.

\section{THE AUTHOR'S CAVEAT AND QUERIES}

(i) Be warned about misleading diagrams. To obtain nonDesarguesian $\mathrm{PG}(\mathrm{n})$ 's we must have $\mathrm{n} \geqslant 9$ and examples are known for $\mathrm{n}=9$. The diagrams in this discourse stop short of this value of $n$. However any attempt to constrict star diagrams for $\mathrm{AG}(9)$ 's is bound to turn up something of interest.

(ii) Does every finite affine plane have a star? If so is every point the centre of some star? 
(iii) Do there exist $2-\left(n^{2}, n+1, n\right)$ designs which are not codesigns for any $A G(n)$ ?

(iv) Suppose an $A G(n)$ has two stars; then does the geometry have a non-trivial automorphism? If there is one star does this guarantee a non-trivial automorphism? What sort of automorphisms guarantee stars in an $A G(n)$ ?

(v) Is there an efficient algebraic mechanism for generating supplementary difference sets for co-designs of extendible affine designs?

\section{REFERENCES}

[1] D.R. Breach, The $2-(9,4,3)$ and $3-(10,5,3)$ designs, J. Comb. Theory (A). 27 (1979), 50-63.

[2] P.J. Cameron and J.H. van Lint, Graphs, codes and designs, Lond. Math. Soc. Lecture Notes, 43, C.U.P. (1980).

[3] P. Dembowski, Inversive planes of even order, Bull. Amer. Math. Soc.,69 (1963), 850-4.

[4] P. Dembowski, Möbiusebenen Gerader Ordnung, Math. Ann., 157 (1964), 179-205.

[5] P. Dembowski, Finite geometries, Springer-Verlag, BerlinHeidelberg - New York (1968).

[6] R.H.F. Denniston, Some new 5-designs, Bull. Lond. Math. Soc., 8 (1976), 263-7.

[7] D.R. Hughes, on t-designs and groups, Amer. J. Math., 87 (1965), 761-78.

[8] D.R. Hughes and F.C. Piper, Projective planes, SpringerVerlag, New York - Heidelberg - Berlin (1973).

[9] W.M. Kantor, Dimension and embedding theorems for geometric lattices, J. Comb. Theory (A), 17 (1974), 173-95.

[10] H. Lüneberg, Die Suzukigruppen and ihre Geometrien, Lecture Notes in Mathematics, 10, Springer-Verlag, BerlinHeidelberg - New York, (1965).

[11] V. Pless, Symmetry codes over GF(3) and new 5-designs, J. Comb. Theory, 12 (1972), 119-42.

[12] E. Witt, Die 5-fach transitiven Gruppen van Mathieu, Abh. Math. Seminar Hamburg, 12 (1938), 256-64.

[13] E. Witt, Über Steineresche Systeme Abh. Math. Seminar Hamburg 12 (1938), 265-275. 


\section{MATHEMATICAL RESEARCH REPORTS}

\section{University of Canterbury}

1. (November 1976) BRYANT, P.J. (Reprinted March 1978)

Permanent wave structures on an open two layer fluid.

2. (December 1976) WLIR, G.J.

Conformal Killing tensors in reducible spaces.

3. (January 1977) WEIR, G.J. \& KSRR, R.P.

Diverging type-D metrics.

4. (February 1977) KERR, R.P.\& WIISON, W.B.

Singularities in the Kerr-Schild metrics.

5. (May 1977) PETSister, G.M. Algebras for matrix limitation.

6. (August 1977) GRAY, A.G.

Topological methods in second order arithmetic.

7. (September 1977) WAYLIN, P.C. Green functions in the early universe.

8. (November 1977) BEAISON, R.K. The asymptotic cost of Lagrange interpolatory side conditions in the space $\mathrm{C}(\mathrm{T})$.

9. (November 1977) BEATSON, R.K. The degree of monotone approximation.

10. (November 1977) BRiACII, D.R. On the non-existence of $5-(24,12,6)$ and $4-(23,11,6)$ designs.

11. (tebruury 1978) BRYANT, P.J. \& LAING, A.K. Permanent wave structuros and resonant triads in a layered fluid.

12. (August 1978) BALL, R.D. Morse theory with applications and extensions.

13. (September 1978) BRYANW, P.J. Nonlinear wave groups in deep water.

14. (September 1978) BRisACI, D.R. Some $2-(2 n+1, n, n-1)$ designs with multiple extensions.

15. (October 1980) CHOW, SHUE-SUM Singular value decomposition and its applications.

16. (January 1981) BREACH, D.R. Some Remarks on a Family of t-designs.

17. (August 1981 ) BRHACH, D.R. Star Gazing in Affine Planes. (Reprinted December 1981)

18. (November 1981) BRYANT, P.J. Short Wave Groups in Deep Water.

19. (November 1981) LEVI, I., WOOD, G.R., and SULLIVAN, R.P. Automorphisms of Baer-Levi Semigroups. 